\title{
BSML: A binding schema markup language for data interchange in problem solving environments ${ }^{1}$
}

\author{
Alex Verstak ${ }^{\mathrm{a}}$, Naren Ramakrishnan ${ }^{\mathrm{a}}$, Layne T. Watson ${ }^{\mathrm{a}}$, Jian He ${ }^{\mathrm{a}}$, Clifford A. Shaffer ${ }^{\mathrm{a}}$, \\ Kyung Kyoon Bae ${ }^{\mathrm{b}}$, Jing Jiang ${ }^{\mathrm{b}}$, William H. Tranter ${ }^{\mathrm{b}}$ and Theodore S. Rappaport ${ }^{\mathrm{b}}$ \\ ${ }^{\mathrm{a}}$ Department of Computer Science, ${ }^{\mathrm{b}}$ Bradley Department of Electrical and Computer Engineering, Virginia \\ Polytechnic Institute and State University, Blacksburg, Virginia 24061, USA \\ E-mail: naren@cs.vt.edu
}

\begin{abstract}
We describe a binding schema markup language (BSML) for describing data interchange between scientific codes. Such a facility is an important constituent of scientific problem solving environments (PSEs). BSML is designed to integrate with a PSE or application composition system that views model specification and execution as a problem of managing semistructured data. The data interchange problem is addressed by three techniques for processing semistructured data: validation, binding, and conversion. We present BSML and describe its application to a PSE for wireless communications system design.
\end{abstract}

\section{Introduction}

Problem solving environments (PSEs) are high-level software systems for doing computational science. A simple example of a PSE is the Web PELLPACK system [20] that addresses the domain of partial differential equations (PDEs). Web PELLPACK allows the scientist to access the system through a Web browser, define PDE problems, choose and configure solution strategies, manage appropriate hardware resources (for solving the PDE), and visualize and analyze the results. The scientist thus communicates with the PSE in the vernacular of the problem, 'not in the language of a particular operating system, programming language, or network protocol' [16]. It is 10 years since the goal of creating PSEs was articulated by an NSF workshop (see [16] for findings and recommendations). From providing highlevel programming interfaces for widely used software libraries [22], PSEs have now expanded to diverse ap-

\footnotetext{
${ }^{1}$ The work presented in this paper is supported in part by National Science Foundation grants EIA-9974956, EIA-9984317, and EIA0103660
}

plication domains such as wood-based composites design [18], aircraft design [17], gas turbine dynamics simulation [15], and microarray bioinformatics [4].

The basic functionalities expected of a PSE include supporting the specification, monitoring, and coordination of extended problem solving tasks. Many PSE system designs employ the compositional modeling paradigm, where the scientist describes data-flow relationships between codes in terms of a graphical network and the PSE manages the details of composing the application represented by the network. Compositional modeling is not restricted to such model specification and execution but can also be used as an aid in performance modeling of scientific codes [2] (model analysis).

We view model specification and execution as a data management problem and describe how a semistructured data model can be used to address data interchange problems in a PSE. Section 1.1 presents a motivating PSE scenario that will help articulate needs from a data management perspective. Section 2 elaborates on these ideas and briefly reviews pertinent related work. In particular, it identifies three basic levels of functionality - validation, binding, and conversion - 
at which data interchange in application composition can be studied. Sections 4, 5, and 6 describe our specific contributions along these dimensions, in the form of a binding schema markup language (BSML). Section 7 outlines how these ideas can be integrated within an existing PSE system design. A concluding discussion is provided in Section 8. Aspects of the scenario described next will be used throughout this paper as running examples.

\subsection{Motivating example}

$\mathrm{S}^{4} \mathrm{~W}$ (Site-Specific System Simulator for Wireless system design) is a PSE being developed at Virginia Tech. $\quad S^{4} \mathrm{~W}$ provides deterministic electromagnetic propagation and stochastic wireless system models for predicting the performance of wireless systems in specific environments, such as office buildings. $\mathrm{S}^{4} \mathrm{~W}$ is also designed to support the inclusion of new models into the system, visualization of results produced by the models, integration of optimization loops around the models, validation of models by comparison with field measurements, and management of the results produced by a large series of experiments. $S^{4} \mathrm{~W}$ permits a variety of usage scenarios. We will describe one scenario in detail.

A wireless design engineer uses $\mathrm{S}^{4} \mathrm{~W}$ to study transmitter placement in an indoor environment located on the fourth floor of Durham Hall at Virginia Tech. The engineering goal is to achieve a certain performance objective within the given cost constraints. For a narrowband system, power levels at the receiver locations are good indicators of system performance. Therefore, minimizing the (spatial) average shortfall of received power with respect to some power threshold is a meaningful and well defined objective. The major cost constraints are the number of transmitters and their powers. Different transmitter locations and powers yield different levels of coverage. The situation is more complicated in a wideband system, but roughly the same process applies. A wideband system includes extra hardware not present in a narrowband system and the performance objective is formulated in terms of the bit error rate (BER), not just the power level.

The first step in this scenario is to construct a model of signal propagation through the wireless communications channel. $\mathrm{S}^{4} \mathrm{~W}$ provides ray tracing as the primary mechanism to model site-specific propagation effects such as transmission (penetration), reflection, and diffraction. The second step is to take into account antenna parameters and system resolution. These two steps are often sufficient to model the performance of a narrowband system. If a wideband system is being considered, the third step is to configure the specific wireless system. Parameters such as the number of fingers of the rake receiver and forward error correction codes are considered at this step. $\mathrm{S}^{4} \mathrm{~W}$ provides a Monte-Carlo simulation of a WCDMA (wideband code division multiple access) family of wireless systems. In either case, the engineer configures a graph of computational components as shown in Fig. 1. The ovals correspond to computational components drawn from a mix of languages and environments. Hexagons enclose input and output data. Aggregation is used to simplify the interfaces of the components to each other and to the optimizer. In Fig. 1, rectangles represent aggregation. The propagation model is a component that consists of three connected subcomponents: triangulation, space partitioning, and ray tracing. Similarly, the wireless system model consists of (roughly) three components: data encoding, channel modeling, and signal decoding. All three steps are further aggregated into a complete site-specific system model. This model is then used in an optimization loop. The optimizer changes transmitter parameters (all other parameters remain fixed) and receives feedback on system performance.

For a given environment definition in AutoCAD, the triangulation and space partitioning components are used to reduce the number of geometric intersection tests that will be performed by the ray tracer. Several iterations over space partitioning are necessary to achieve acceptable software performance. However, once the objective (an average of ten triangles per voxel) is met, the space partitioning can be reused in all future experiments with this environment. The engineer then configures the ray tracer to only capture reflection and transmission (penetration) effects. Although diffraction and scattering are important in indoor propagation [5], these phenomena are computationally expensive to model in an optimization loop. The triangulation and space partitioning codes are meant for serial execution, whereas the ray tracer and the Monte Carlo wireless system models run on a 200 node Beowulf cluster of workstations. Post processing is available in both serial and parallel versions. The ray tracer and the post processor are written in C, whereas the WCDMA simulation is available in Matlab and Fortran 95 versions.

A series of experiments is performed for various choices of antenna patterns, path loss parameters (influenced by material properties), and WCDMA system parameters. The predicted power delay profiles (PDPs) 


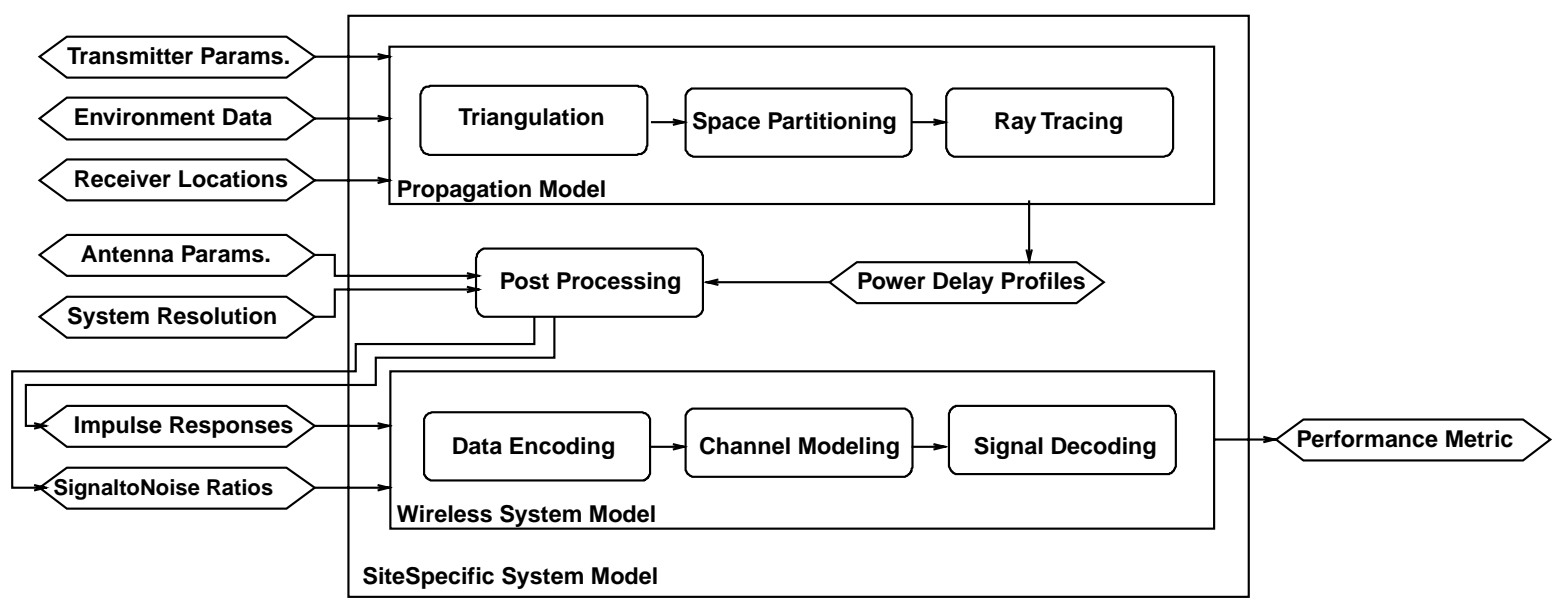

Fig. 1. A site-specific system model in $\mathrm{S}^{4} \mathrm{~W}$. The system model consists of a propagation model, an antenna model (post processing), and a wireless system model.

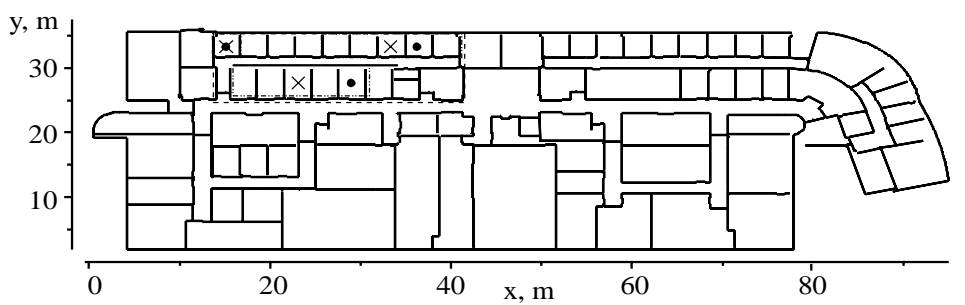

Fig. 2. Optimizing placement of three transmitters to cover eighteen rooms and a corridor bounded by the box in the upper left corner. The bounds for the placement of three transmitters are drawn with dotted lines. The initial transmitter positions are marked with crosses. The optimum coverage transmitter positions are marked with dots.

are then compared with the measurements from a channel sounder and the predicted bit error rates are compared with the published data. The parameters of the propagation model are calibrated for various locations. The validated propagation and wireless system models are finally enclosed in an optimization loop to determine the locations of transmitters that will provide adequate performance for a region of interest. The optimizer, written in Fortran 95, uses the DIviding RECTangles (DIRECT) algorithm of Jones et al. [19]. The parameters to the optimization problem and the optimal transmitter placement are depicted in Fig. 2. The optimizer decided to move the transmitter in the upper right corner one room to the right of its initial position and the transmitter in the lower left corner two rooms to the right of its initial position.

What requirements can we abstract from this scenario and how can they be flexibly supported by a data model? We first observe the diversity in the computational environment. Component codes are written in different languages and some of them are meant for parallel execution. In a research project such as
$\mathrm{S}^{4} \mathrm{~W}$, many components are under active development, so their I/O specifications change over time. Second, the interconnection among components is also flexible. Optimizing for power coverage and optimizing for bit error rate, while having similar motivations, require different topologies of computational components. Third, since different groups of researchers are involved in the project, there exists significant cognitive discordance among vocabularies, data formats, components, and even methodologies. For example, ray tracing models represent powers in a power delay profile in $\mathrm{dBm}$ (log scale). However, WCDMA models work with a normalized linear scale impulse response and an aggregate called the 'signal-to-noise ratio'. Also, there is more than one way of calculating the signal-to-noise ratio. Since antennas generate noise that depends on their parameters, detailed antenna descriptions are necessary to calculate this ratio. However, researchers who are not concerned with antenna design seldom model the system at this level of detail. The typical practice is to use a fixed noise level in the calculations. Simulations 
of wireless systems abound in such approximations, ad hoc conversions, and simplifying assumptions.

\section{PSE requirements for data interchange}

Culling from the above scenario, we arrive at a more formal list of data interchange requirements for application composition in a PSE. The PSE must support:

1. components in multiple languages (C, FORTRAN, Matlab, SQL);

2. changes in component interfaces;

3. changes in interconnections among components;

4. automatic unit conversion in data-flows;

5. user-defined conversion filters;

6. composition of components with slightly different interfaces; and

7. stream processing.

The reader might be surprised that SQL is listed alongside FORTRAN, but both languages are used in $\mathrm{S}^{4} \mathrm{~W}$. Experiment simulations are written in procedural languages, while experiment data is stored in a relational database. Thus, developing a system that integrates with the PSE environment requires more than the ability to link scientific computing languages. It involves overcoming the impedance mismatch between languages developed for fundamentally different purposes.

The last requirement above - stream processing refers to processing data as soon as it is read from an input stream, as opposed to waiting for the end of the stream, and subsequently processing all the data at once. This often neglected technical requirement is related to composability - the ability to create arbitrary component topologies. As data interchange is pushed deeper into the computation, the unit of data granularity needs to become correspondingly smaller. The optimization loop is a good example of fine data granularity. We cannot accumulate all transmitter parameters over all iterations and later convert them to the format required by the simulation inside the loop, because transmitter parameters generated by the optimizer depend on the feedback computed by the simulation. Each block of transmitters must be processed as soon as it is available. Likewise, each value of the objective function must be made available to the optimizer before it can produce the next block of transmitters. Usability dictates a similar requirement. Since some models are computationally expensive (e.g., those meant for parallel execution), incremental feedback should be pro- vided to the user as early as possible. The stream processing requirement improves composability and usability, but limits conversions to being local. Global conversions (e.g., XSLT [13]) cannot be performed because they assume that all the data is available at once.

While the requirements point to a semistructured data model, no currently available data management system supports all forms of PSE functionality. This paper presents the prototype of such a system in the form of a markup language. Observe that all of the above requirements are summarized by three standard techniques for working with semistructured data - validation, binding, and conversion. Validation establishes data conformance to a given schema. It is a prerequisite to most of the requirements. Binding refers to integrating semistructured data with languages that were designed for different purposes (requirement 1). Conversion (transformation) takes care of requirements 2-6. Given two slightly different schemas, it is possible to generate an edit script [11] that converts data instances from one schema to another. Requirement 7 dictates that all such conversions must be local.

\subsection{Related work}

While research in PSEs covers a broad territory, the use of semistructured data representations in computational science is not established beyond a few projects. Therefore, we only survey standard XML technologies and PSE-like systems that make (some) use of semistructured data. It would be unfair to review some of these systems against PSE data interchange requirements. Instead, our evaluation is based on how well these systems support validation, binding, conversion, and stream processing.

Specific XML technologies for document processing are easy to classify in terms of our framework. Schema languages (e.g., RELAX NG [12]) deal with validation and, possibly, binding. Transformation languages (e.g., XSLT [13]) deal with conversion. Several properties of these technologies hinder their direct applicability to a PSE setting. First and foremost, these technologies do not work with streams of data. Sophisticated schema constraints and complex transformations can require buffering the whole document before producing any output. Second, transformation languages are simply vehicles for applying edit scripts. They cannot be used to create edit scripts. Since our conversions are local, edit script application is trivial, but edit script creation is not.

Four major flavors of PSE-like projects that use semistructured data representations can be identified: 
1. component metadata projects;

2. workflow projects;

3. scientific data interchange projects; and

4. scientific data management projects.

Projects in the first category use XML to store IDLlike (interface definition language) component descriptions and miscellaneous component execution parameters. An example of such a project is CCAT [9], which is a distributed object oriented system. CCAT also uses XML for message transport between components, so we say that it provides an $\mathrm{OO}$ binding. The second category of projects augments component metadata with workflow specifications. For example, GALE [8] is a workflow specification language for executing simulations on distributed systems. Unlike CCAT, GALE provides XML specifications for some common types of experiments, such as parameter sweeps (CCAT uses a scripting language for workflow specification). However, GALE does not use XML for component data. Both the component metadata and workflow projects use XML to encode data that is not semistructured. Their use of XML is not dictated by the need for automatic conversion. Neither generic binding mechanisms nor conversion are provided by these projects.

The latter two groups of projects use XML for application data, not component metadata. Representatives of the scientific data interchange group develop flexible all-encompassing schemas for specific application domains. For example, CACTUS [7] deals with spatial grid data. CACTUS's schema is complex enough to be considered semistructured and this project recognizes the need for conversion filters. However, it does not provide multiple language support and, more importantly, does not accommodate changes in the schema. CACTUS's conversion filters aim at code reuse, not change management. This project has $\mathrm{OO}$ binding and manual conversion (the sequence of conversions is not determined automatically). Complexity of the data format precludes stream processing.

Perhaps the most relevant group of projects for our purposes involves the scientific data management community. Especially interesting are the projects in rapidly evolving domains, such as bioinformatics. DataFoundry $[1,14]$ provides a unifying database interface to diverse bioinformatics sources. Both the data and the schema of these sources evolve quickly, so DataFoundry has to deal with change management - by far more complex change management than the kind we consider here. However, DataFoundry only provides mediators for database access. It does not integrate with simulation execution. This system takes full ad- vantage of conversion, but provides only an SQL binding. Introducing bindings for procedural languages would involve significant changes to DataFoundry.

Table 1 summarizes related work. It turns out that no known PSE-like system takes full advantage of both binding and conversion. XML technologies for validation and binding are well established, but XML transformation technologies do not support PSE-style conversion. Very few systems can integrate with a PSE execution environment because most of them do not meet the stream processing requirement. This paper develops a system that satisfies all of our data interchange requirements. The next section summarizes the contributions made by our approach and also introduces relevant background material. The following three sections describe our handling of validation, binding, and conversion. System integration is outlined in Section 7.

\section{In this paper}

As mentioned earlier, our specific contributions are in the form of a markup language called BSML. BSML provides expressive access to objects and streams for managing the execution environment of a PSE. It should be remarked that BSML is not a data format masquerading as a markup language, or even a highlevel abstraction of a programming environment. It is meant to be a vehicle to capture assumptions about data interchange happening in a PSE. Suitably defined BSML schemas allow the programmer to describe mappings from internal representations to the execution environment. These mappings are used to perform validation, binding, and conversion functions. Validation is achieved by ensuring that new documents (describing PSE objects) conform to BSML schemas. Binding is achieved by inserting special markup tags that describe how PSE objects should be interpreted in an underlying environment. Conversion is motivated by relating BSML schemas. Specifically, we create a schema that describes one data format but performs the bindings of another data format.

The novelty of our work is a careful integration of the relevant concepts - parsing theory as it relates to attribute grammars, realistic PSE settings, and a markup language as a mechanism to capture assumptions. A core set of algorithmic ideas transcend all of BSML's capabilities. These specific ideas include: (i) relating stream processing requirements of PSEs to predictive parsing theory, (ii) studying how PSE requirements for binding manifest in attribute grammars, including 
Table 1

A survey of PSE-like systems and XML technologies. The binding row shows that most systems support only one paradigm. Only DataFoundry fully supports conversion. Other systems either provide a library of conversion primitives and leave their composition up to the user (CACTUS) or do not recognize the need for conversion at all (CCAT). No system or technology fully supports validation, binding, and conversion. Most systems and technologies cannot dynamically process streams of data

\begin{tabular}{lcccccc}
\hline & CCAT & GALE & CACTUS & DataFoundry & RELAX NG & XSLT \\
\hline Validation & $\sqrt{ }$ & & $\sqrt{ }$ & $\sqrt{ }$ & $\sqrt{ }$ & \\
Binding & OO & & OO & SQL & OO & \\
Conversion & & & manual & $\sqrt{ }$ & & manual \\
Stream Processing & $\sqrt{ }$ & & & & & \\
\hline
\end{tabular}

their effect on predictive parsers, and (iii) using schema transformations to provide conversion and change management functionality. Our work is one of the first efforts to systematize the creation of conversion facilities in a PSE.

\subsection{Some pertinent background}

We begin by reviewing some pertinent background in the areas of markup languages and parsing theory. Markup languages, like XML, HTML, and SGML, use a tagged structure to describe documents. While the types and intended semantics of tags are fixed in a language like HTML, tags in XML do not have any pre-defined meaning. This allows us to rapidly prototype domain-specific markup languages (like BSML) and use document processing tools to harness descriptions in such languages. Ultimately, this availability of readymade software is what steers scientific computing researchers to a markup language-based solution.

One typical use of a markup language is for defining data formats. For instance, we can define a markup language for describing time series data. Domains such as bioinformatics abound in such markup languages. BSML's approach is to employ tags that will help realize data interchange functionality. There are even projects that encapsulate a complete ontology in a markup language!

Documents in a markup language can be displayed, interpreted, and reasoned about in simple ways. For instance, a web browser uses the $\langle\mathrm{B}\rangle \ldots</ \mathrm{B}\rangle$ tag structure in a HTML document to recognize when to render text in bold. Similarly, we can assign any suitable interpretation to a markup language in a PSE setting.

A markup language can be defined by its DTD (Document Type Definition) which declares what a well formed document should look like. Among other things, the DTD helps validate new documents, to see if they adhere to the markup specification. Other tools use DTDs to automatically generate parsers for inter- preting documents. XML Schema is a newer approach for schema definition of XML documents and is widely believed to eventually supersede DTDs. BSML can actually be thought of as a schema language specifically designed for data interchange in PSEs.

Two technologies that are especially relevant here are DOM and SAX. DOM (Document Object Model) is an object model that uses a tree structure to represent an XML document. This internal tree structure can then be navigated and manipulated to provide many facilities, e.g., searching the tree for the occurence of a given string, or rearranging the tree structure to produce a new document. The contrasting approach, SAX, is an event-based technology that relates parsing events back to an application, which can then use them to implement specific functionality. Many parsing tools use either or both these approaches. The reader is referred to introductory resources such as [10] for more details.

Besides markup language basics, this paper assumes background knowledge of grammars and computer languages, especially as encountered in a compilers course. The most important concepts are LL grammars and the construction of predictive parsing tables. For our purposes, an LL grammar is one that supports iterative and incremental parsing of input and as we will show, this is a necessary pre-requisite to achieve data interchange funtionality. The first ' $L$ ' denotes a 'leftto-right' scan and the second ' $L$ ' denotes that we are performing a leftmost derivation. We will devote considerable attention to LL(1) grammars which are LL with only one symbol of lookahead. These concepts are well covered in [3].

\section{Validation}

The first function we study, validation, establishes conformance of a data instance to a given schema. It is a prerequisite to binding and conversion. (This def- 
inition of validation is a small part of the process of validation in a PSE, which is concerned with the larger issue of a model being appropriate to solve a given problem; but, it suffices for the purpose of this paper.) The schemas for PSE data are easy to obtain since computational science traditionally uses rigid data structures, not loosely formatted documents. Describing the data structures in terms of schemas has several benefits. First, language-neutral schemas allow for interoperability between different languages (see requirement 1 in the previous section). Second, schemas facilitate database storage and retrieval. Third, appropriate schemas help assign interpretations to various data fields. It is such interpretation that makes automatic conversion possible (requirements 2-6).

What kind of validation is appropriate for PSE data? Requirement 7 calls for the most expressive schema language that can be parsed by a stream parser. In other words, we are looking for a schema language that can be defined in terms of an LL(1) grammar [3]. (The LR family of grammars is more expressive, but LR parsers do not follow stream semantics.) Therefore, a predictive parser generated for a given schema can validate a data instance. This section describes a schema language (BSML) appropriate for a PSE and the steps for building a parser generator for this language. We present an example, an informal overview of BSML features, and a formal definition for a large subset of BSML in terms of a context-free grammar. Further, predictive parser generation is outlined and grammar transformations specific to BSML are described in detail. Finally, we show that BSML is strictly less expressive than LL(1) grammars.

Let us start with an example. Figures 3 and 4 depict a (simplified) schema for an octree environment decomposition. (Figure 3 describes it in XML notation while Fig. 4 uses a non-XML format that will be useful for describing some functionalities of BSML). This is the most complex schema in $\mathrm{S}^{4} \mathrm{~W}$, not counting the schema for the schema language itself. An octree consists of internal and leaf nodes that delimit groups of triangles. Recall from Section 1.1 that this grouping is used to limit the intersection tests in ray tracing. The nested structure of an octree maps nicely into an XML tree. Since many components work with lists of triangles, there is a separate schema for a list of triangles. As the example shows, the features of BSML closely resemble those of other schema languages, such as RELAX NG. The only noticeable difference is the presence of units in the definitions of primitive types. Units will be useful for certain types of conversions. Figure 5 shows an LL(1) grammar generated from the octree schema. This grammar is then annotated with binding code and used to generate a parser for octree data. The parser can be linked with a parallel ray tracer written in $\mathrm{C}$.

The DTD for the current version of BSML is given in Appendix 8. The schema language describes primitive types and schemas. There are four base primitive types: integer, string, (IEEE) double, and boolean. Users can derive their own primitive types by range restriction. User-derived types usually have domain-specific flavor, such as coordinates and distances in the example above. We do not support more complicated primitive types, such as dates and lists, because each PSE component treats them differently. Schemas consist of four building blocks: elements, sequences, selections, and repetitions. Strictly speaking, repetitions can be expressed as selections and sequences, but they are so common that they deserve special treatment. Derivation of schemas by restriction is not supported, but derivation by extension can be implemented via inter-schema references. Mixed content is not supported because it is only used for documentation. Instead, BSML supports a wildcard content type. The contents of this type matches anything and is delivered to the component as a DOM tree [6]. We do not support referential integrity constraints because they can delay binding and thus break requirement 7 . There is no explicit construct for interleaves. In some ways, interleaves are handled by the conversion algorithm. In other words, BSML is a simple schema language that incorporates most common features that are useful in a PSE.

Parser generation for a BSML schema follows the standard steps from compiler textbooks [3]:

1. convert the schema to an LL(1) grammar,

2. eliminate empty productions and self-derivations,

3. eliminate left recursion,

4. perform left factoring,

5. perform miscellaneous cleanup (described in detail below),

6. compute a predictive parsing table, and

7. generate parsing code from the table.

The only steps specific to this schema language are generating an LL(1) grammar (step 1) and miscellaneous cleanup (step 5). Since grammars have been in use for a long time, it is pertinent to define BSML semantics in terms of how the schemas are converted to grammars. The terminals are defined by SAX events [10]. The start of element and end of element events are denoted $s$ (name) and $e$ (name), respectively, where name is element name. We omit 


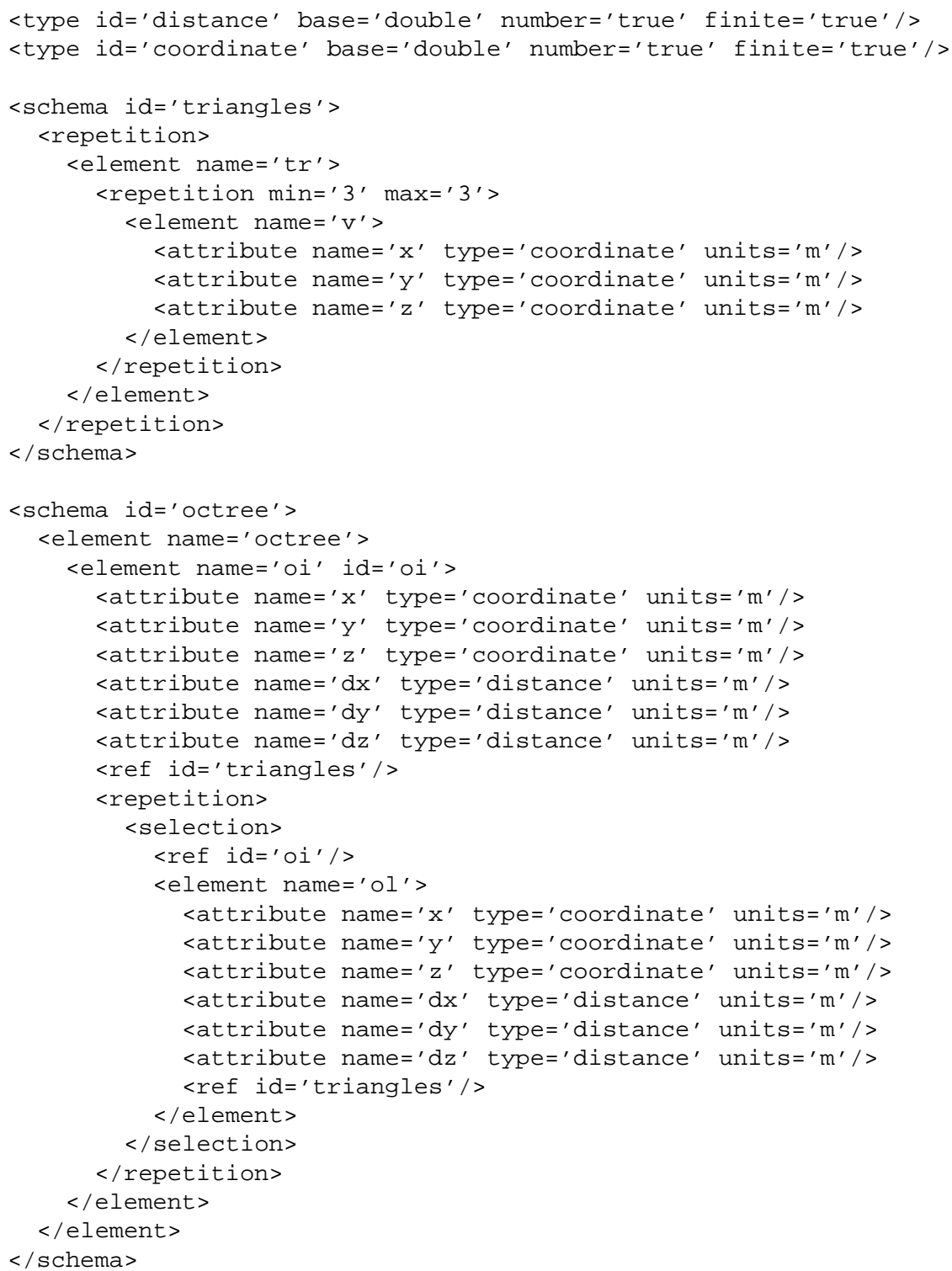

Fig. 3. BSML schemas for an octree decomposition of an environment, in XML notation. 'tr' stands for a triangle, 'v' stands for a vertex, 'oi' stands for an internal node, and 'ol' stands for a leaf.

the attributes for simplicity, but BSML supports them in an obvious way. Further, we assume that the SAX parser inlines external entity references. Character data is accumulated until the next start of element or end of element event and delivered as a $d$ (base, min, max, number, finite, units) terminal, abbreviated as $d$ (see Appendix 8 for $d$ 's attributes). Generated code checks character data conformance to the type constraints. This definition of $d$ is appropriate since BSML does not support selections based on the type of character data.
One root non-terminal is initially generated for each schema block (element, sequence, selection, repetition), each reference to a primitive type, and each string of user code. We denote non-terminals by capital letters, the start non-terminal by $S$, the empty string by $\epsilon$, and the root non-terminals generated for the children of each schema block by $X_{1}, X_{2}, \ldots, X_{n}, n \mathfrak{e} 0$. Further, lower-case Greek letters denote (possibly empty) sequences of terminals, non-terminals, and, in the next section, user codes. With this notation in mind, the definition of BSML is in Fig. 6 (more details follow in fu- 


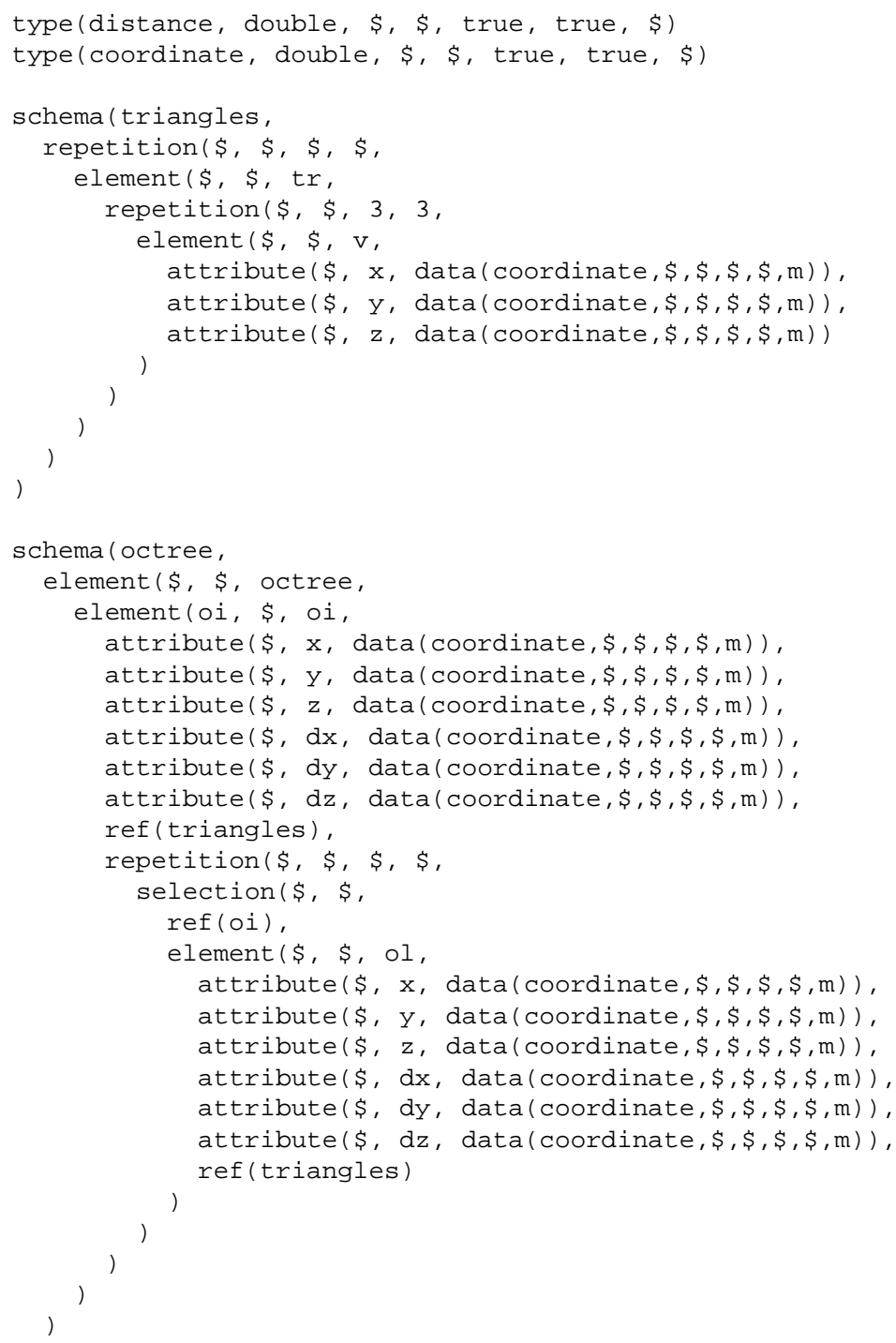

Fig. 4. BSML schemas from Fig. 3 in a non-XML notation. \$ stands for a missing value, i.e., a suitable default value is supplied by BSML software.

ture sections). We slightly deviate from a context-free grammar to allow for the constraints on the number of repetitions (see next section). To reiterate, a grammar generated from a schema according to this definition will undergo several standard equivalence transformations before a grammar of the form shown in Fig. 5 is obtained.

The purpose of miscellaneous cleanup is to reduce the number of non-terminals in the grammar. These ad-hoc rewritings do not guarantee that the resultant grammar is minimal in any strict sense. Instead, they address some inefficiencies that other steps are likely to introduce. These cleanup steps were also chosen such that if the grammar were LL(1) before cleanup, it would remain LL(1) after cleanup. The grammars shown in this paper have undergone two cleanup rewritings. Each rewriting is applied until no further rewriting is possible.

1. Maximum length common suffixes are factored out. $\beta \neq \epsilon$ is the maximum length common suffix of a non-terminal $A \neq S$ if (a) all of $A$ 's productions have the form $A \rightarrow \alpha_{i} \beta, 1 \leq i \leq n$, (b) $\beta$ is of maximum length, and (c) neither $\beta$ nor any $\alpha_{i}$ contain $A$. If $n=1, A$ is eliminated from the grammar and all occurrences of $A$ in the grammar 


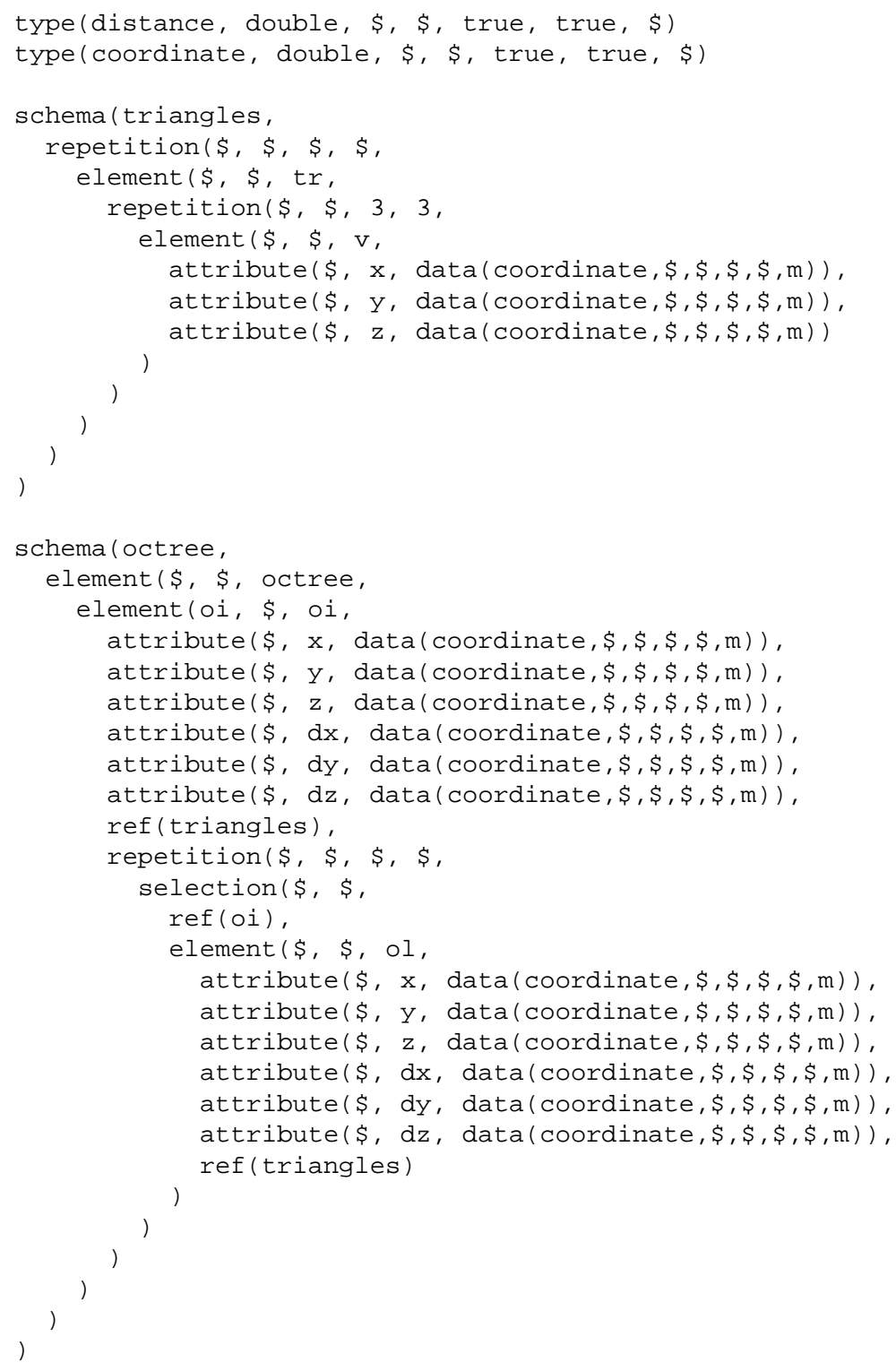

Fig. 5. LL(1) grammar corresponding to the octree schemas in Figures 3 and 4. Attributes are omitted for simplicity. Patterns of the form $\{c\}$ will be explained in the next section (they are related to repetitions). Non-terminals $T, T^{h}$, and $V$ are related to triangles; others are related to octree decomposition of a set of triangles.

are replaced with $\beta$ ( $\alpha_{1}=\epsilon$ because $\beta$ is of maximum length). We call such non-terminals trivial. Trivial non-terminals are often introduced by schema-to-grammar conversion rules. If $n>1$, all occurrences of $A$ on the right-hand sides of all grammar productions are replaced with $A \beta$ and the suffix $\beta$ is deleted from all of $A$ 's productions. The purpose of this rewriting is to uncover duplicate non-terminals for the next step.

2. Only one of any two duplicate non-terminals is retained. Two non-terminals $A \neq B$ are duplicate if whenever $A \rightarrow \alpha$ is in the grammar, $B \rightarrow \alpha$ is also in the grammar, and vice versa. $A$ is eliminated if $A \neq S, B$ is eliminated otherwise. This definition is weak, e.g., $A$ and $B$ are not considered duplicate if $A \rightarrow \alpha A \beta$ and $B \rightarrow$ $\alpha B \beta$ are in the grammar. However, it suffices for our purposes.

The expressive power of LL(1) grammars is well known. In practice, the limiting factor is not that the 


\begin{tabular}{|c|c|c|c|}
\hline element (id, opt, name, $B_{1}, B_{2}, \ldots, B_{n}$ ) & $E$ & $\rightarrow$ & $s$ (name) $, X_{1}, X_{2}, \ldots, X_{n}, e$ (name) \\
\hline & $E$ & $\rightarrow$ & $\epsilon$ if opt \\
\hline sequence (id, opt, $\left.B_{1}, B_{2}, \ldots, B_{n}\right)$ & $Q$ & $\rightarrow$ & $X_{1}, X_{2}, \ldots, X_{n}$ \\
\hline & $Q$ & $\rightarrow$ & $\epsilon$ if opt \\
\hline selection(id, opt, $\left.B_{1}, B_{2}, \ldots, B_{n}\right)$ & $L$ & $\rightarrow$ & $X_{1}$ \\
\hline & $L$ & $\rightarrow$ & $X_{2}$ \\
\hline & & & $\cdots$ \\
\hline & $L$ & $\rightarrow$ & $X_{n}$ \\
\hline & $L$ & $\rightarrow$ & $\epsilon$ if opt \\
\hline repetition $\left(i d\right.$, opt, $\left.\min , \max , B_{1}, B_{2}, \ldots, B_{n}\right)$ & $R$ & $\rightarrow$ & $\{B\}, X_{1}, X_{2}, \ldots, X_{n},\{A\}, R^{\prime},\{E\}$ \\
\hline & $R^{\prime}$ & $\rightarrow$ & $X_{1}, X_{2}, \ldots, X_{n},\{A\}, R^{\prime}$ \\
\hline & $R^{\prime}$ & $\rightarrow$ & $\epsilon$ \\
\hline & $R$ & $\rightarrow$ & $c$ if opt or $\min =0$ \\
\hline data(base, min, max, number, finite, units) & $D$ & $\rightarrow$ & $d($ base, min, mar, number, finite, units) \\
\hline code $(c)$ & $C$ & $\rightarrow$ & $\{c\}$ \\
\hline
\end{tabular}

Fig. 6. L-attributed definition of BSML. Schema primitives, in a non-XML notation, are on the left (see Figure 4 for an example) and their translations to grammar productions are on the right. $B_{1}, B_{2}, \ldots, B_{n}$ are the children of the schema block and $X_{1}, X_{2}, \ldots, X_{n}$ are the root non-terminals generated for $B_{1}, B_{2}, \ldots, B_{n}$, respectively. opt is a boolean block attribute; true means that the block is optional. $\{B\},\{A\},\{E\}$, and $\{c\}$ are binding codes explained in the next section. References to schema blocks (denoted by ref $(i d)$ ) are replaced with root non-terminals of the blocks being referenced. Definitions related to XML attributes are omitted.

grammar is LL(1), but that the grammar is annotated with user codes. The next section gives two examples of grammars that are not convertible to LL(1) because binding codes are present. A more interesting question is how the expressive power of LL(1) grammars compares to the expressive power of BSML. It is easy to see that BSML can express a proper subset of LL(1) grammars. For example, $S \rightarrow s(x), e(y)$ is a valid LL(1) grammar, but BSML cannot express it since no XML document that conforms to this grammar is wellformed.

Observation 1 Consider a subset of BSML that excludes repetitions and user codes. We say that BSML can express a grammar $G$ if a predictive parser generated from some schema in this restricted subset of BSML can recognize precisely the language $L(G)$. Clearly, $B S M L$ cannot express any grammar $G$ that is not $L L(1)$ (by construction of the predictive parser). Further, BSML cannot express an LL(1) grammar $G$ unless:

1. if $d_{1}$ and $d_{2}$ are data terminals in $G$, then $\forall \alpha, \beta$ : $S \nRightarrow{ }^{+} \alpha, d_{1}, d_{2}, \beta$ (data is atomic),

2. if $d$ is a data terminal and $S \Rightarrow^{+} \alpha, d, \beta$ is a derivation in $G$, then

$\forall x, \gamma:\left(\left[\beta \nRightarrow \nRightarrow^{*} s(x), \gamma\right]\right.$ and $\left[\left(\beta \Rightarrow^{*} e(x), \gamma\right)\right.$ implies $\left.\left.\left(\forall y, \theta: \alpha \nRightarrow \nRightarrow^{*} \theta, e(y)\right)\right]\right)$ (no mixed contents), and

3. if $s(x)$ is a start of element terminal, $g$ is $\epsilon$ or a data terminal, and $S \Rightarrow^{+} \alpha, s(x), \beta$ is a deriva-

$$
\begin{aligned}
& \text { tion in } G \text {, then }\left(\left[\beta \nRightarrow^{*} g\right] \text { and }[(y \neq x) \text { implies }\right. \\
& \left.\left.\left(\forall \gamma: \beta \nRightarrow^{*} g, e(y), \gamma\right)\right]\right) \text {; similarly, if } e(y) \text { is an } \\
& \text { end of element terminal and } S \Rightarrow^{+} \alpha, e(x), \beta \text { is } \\
& \text { a derivation in } G \text {, then }\left(\left[\alpha \nRightarrow^{*} g\right] \text { and }[(x \neq y)\right. \\
& \text { implies } \left.\left.\left(\forall \theta: \alpha \nRightarrow^{*} \theta, s(x), g\right)\right]\right) \text { (proper nesting } \\
& \text { of elements). }
\end{aligned}
$$

The first two restrictions are specific to BSML and easy to relax. However, the last restriction is inherent in any XML schema language. A good schema language cannot describe documents that are not well-formed. These are the necessary conditions, but it is not clear whether or not they are sufficient. We define schemas in terms of the schema language, not in terms of LL(1) grammars, so converting from grammars to schemas is not considered in this paper.

This section provided an overview of BSML features and defined BSML in terms of an 'almost context-free' grammar. We outlined automatic generation of predictive parsers that validate XML documents. Further, we have shown that the descriptive power of BSML is strictly less than that of an LL(1) grammar where the terminals are SAX events. The next section extends validation to perform binding.

\section{Binding}

Binding is a way to integrate semistructured data with languages that were not designed to handle it (re- 
quirement 1). Binding can take several forms, depending on the language. For FORTRAN and $\mathrm{C}$, binding usually means assigning values to language variables and calling user-defined code to process these values (procedural binding). It can also mean writing the data out in a format understood by the component (format conversion). For Matlab and SQL, binding entails generating a script that contains embedded data and processing this script by an interpreter (code generation). The last two kinds of binding can be thought of as XSLT-like transformations.

We implement all three kinds of binding by Lattributed definitions. The schema language is extended by allowing user code to be injected in the schema. Schema languages that provide binding are called binding schema markup languages. This section describes bindings in BSML and gives an example of their use. Further, we show how arbitrary binding codes limit the set of schemas supported by BSML. Predictive parsing cannot handle common prefixes in alternative productions, so standard techniques are used to eliminate such common prefixes. We show that these techniques break when the common prefixes contain binding codes. This limitation is rarely an issue and the problems it causes can be remedied by simple modifications to the schema.

Let $c$ denote an arbitrary string of code. Matching $\{c\}$ means executing code $c$ while consuming no input tokens. No assumptions are made about the nature of $c$. In particular, $c$ can (and usually does) produce side effects, so $A \rightarrow\left\{c_{1}\right\},\left\{c_{2}\right\}$ and $A \rightarrow\left\{c_{2}\right\},\left\{c_{1}\right\}$ can yield different results. A syntax-directed definition is a context-free grammar extended by allowing $\left\{c_{j}\right\}$ on the right-hand sides of productions. For a syntaxdirected definition to be useful in binding, $c_{j}$ must contain references to parts of the document being parsed. We denote such references by $\circ \mathrm{x}$, where $\mathrm{x}$ is the id or the name of some element or attribute. When $x$ refers to an attribute or an element of some primitive type, $\circ \mathrm{x}$ is a value of the attribute or the data contents of the element. The type of $\circ \mathrm{x}$ is determined by the corresponding primitive type. When $\mathrm{x}$ refers to an element of a wildcard type, $\circ \mathrm{x}$ is a DOM tree constructed from all descendants of $x$, including itself. This feature can be used for XHTML [21] documentation. The set of attributes (elements) that are available to code $c$ depends on the placement of $c$ in the syntax-directed definition and the parsing strategy. A syntax-directed definition is $L$-attributed if, for any derivation $S \Rightarrow{ }^{+} \alpha\{c\} \beta$, any $\mathrm{x}$ referenced in $c$ is defined in all derivations of $\alpha$. That is, all attributes (elements) must be defined in a left- to-right scan before they are referenced. L-attributed definitions are easy to implement with an LL(1) parser, but they restrict the set of grammars reducible to LL(1). Luckily, these restrictions are not important in practice.

Figure 7 gives an example binding schema for a PDP (see Section 1.1) and Fig. 8 shows how a parser generated from this schema converts a PDP encoded in XML to a Matlab script. This script will then be executed by an execution manager (see Section 7). The same schema, with different binding code, can convert an XML file to a number of SQL INSERT statements that record the data in a relational database. The semantics of user codes are not limited to printing, so a FORTRAN version of this binding can store the PDP in an array to be processed later. In other words, BSML bindings are compatible with any execution environment that processes streams of data (requirement 7). We use the same approach to convert semistructured data to relational data, Matlab scripts, and $\mathrm{C}$ structures.

The $\{B\},\{A\}$, and $\{E\}$ codes in Fig. 7 are generated for repetitions. They are not necessary for this example, but are required to enforce that each triangle has three vertices in the previous example. $\{B\}$ (begin repetition) initializes the repetition count to zero. Each repetition has its own stack of counts. $\{A\}$ (append) ensures that the maximum allowed number of repetitions is not exceeded. $\{E\}$ (end) checks the minimum number of repetitions. Thus, even simple validation (without binding) is implemented in terms of an L-attributed definition, not just an LL(1) grammar.

Unfortunately, L-attributed definitions make predictive parsing of certain grammars impossible. User codes can prevent elimination of left recursion or left factoring of an L-attributed definition. In the two examples below, grammars induced from the left-attributed definitions by removing all user code can be transformed to LL(1). However, the original L-attributed definitions cannot be transformed to LL(1) without losing the stream semantics of the parser.

Example 1 Consider a left-recursive schema and the corresponding left-recursive grammar (after eliminating trivial non-terminals):

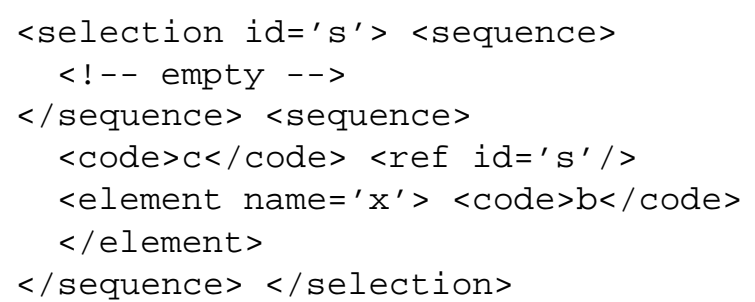




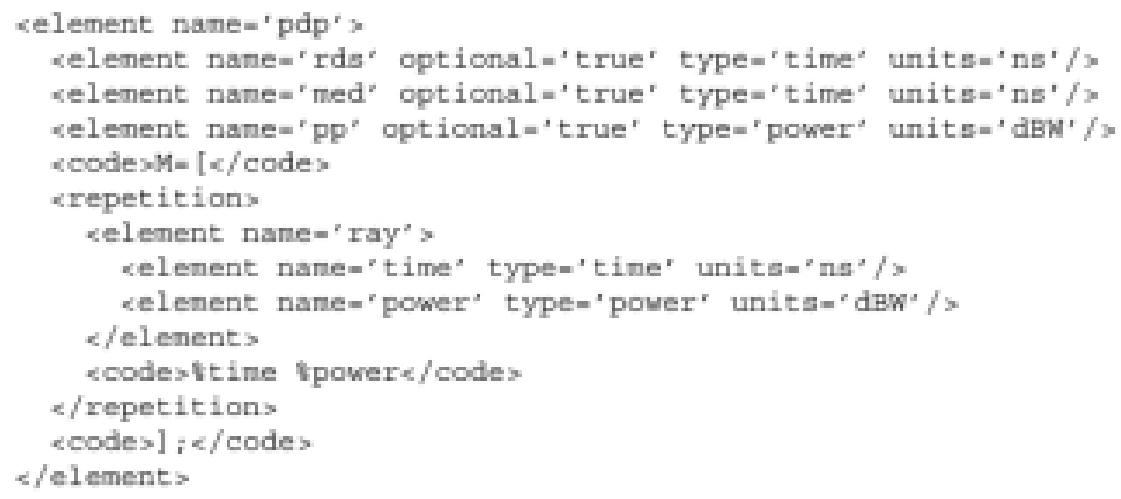

Fig. 7. (top) Binding schema for a power delay profile. $r d s$, med, and $p p$ stand for various optional statistics: rms delay spread, mean excess delay, and peak power. These statistics are ignored in this example. (left) L-attributed definition for a power delay profile. $\{B\},\{A\}$, and $\{E\}$ stand for codes generated by the parser generator to handle repetitions. Otherwise, the meaning of $\{c\}$ is to print string $c$, followed by a new line character, after expanding element references. For clarity, full suffix factoring was not performed, but trivial productions were eliminated. (right) Predictive parsing table for a power delay profile.

$$
\begin{aligned}
& S \rightarrow \epsilon \\
& S \rightarrow\{c\}, S, s(x),\{b\}, e(x)
\end{aligned}
$$

This grammar permits a derivation of the form $S \Rightarrow^{+}\{c\}^{k},(s(x),\{b\}, e(x))^{k}, k>0$. However, code $b$ cannot be executed before $k$ is known since $k$ executions of code c must precede the first execution of code b. Therefore, no LL(1) parser with stream semantics can parse documents that conform to this schema. On the other hand, removing $\{c\}$ from the L-attributed definition yields a grammar that is easily converted to $L L(1)$ :

$$
\begin{aligned}
& S \rightarrow \epsilon \\
& S \rightarrow S, s(x),\{b\}, e(x) \\
& S \rightarrow \epsilon \\
& S \rightarrow s(x),\{b\}, e(x), S
\end{aligned}
$$

This example is easy to generalize.

Observation 2 Consider a set of all productions for a non-terminal $A$. Since any sequence $\left\{c_{1}\right\}\left\{c_{2}\right\}$ can be rewritten as $\{c\}$, where $c=c_{1} c_{2}$, we can uniquely represent this set by

$$
\begin{aligned}
A \rightarrow & \left\{c_{1}\right\} A \alpha_{1}\left|\left\{c_{2}\right\} A \alpha_{2}\right| \cdots \mid \\
& \left\{c_{n}\right\} A \alpha_{n}\left|\beta_{1}\right| \beta_{2}|\cdots| \beta_{m},
\end{aligned}
$$

where no $\beta_{j}, 1 \leq j \leq m$, has a prefix $\{d\} A$. Immediate left recursion can be eliminated from this production without delaying user code execution if and only if

1. $c_{1}=c_{2}=\cdots=c_{n}=\epsilon$ (no user code to the left)

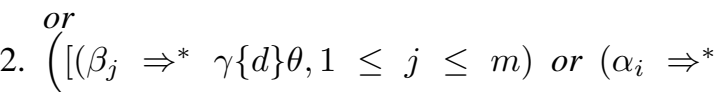
$\gamma\{d\} \theta, 1 \leq i \leq n)]$ implies $(d=\epsilon)$ ) (no user code to the right) and $\left(c_{1}=c_{2}=\cdots=c_{n}\right)$ (same user code to the left).

In all other cases, execution of user code must be delayed until the last $\alpha_{i}$ is matched. 

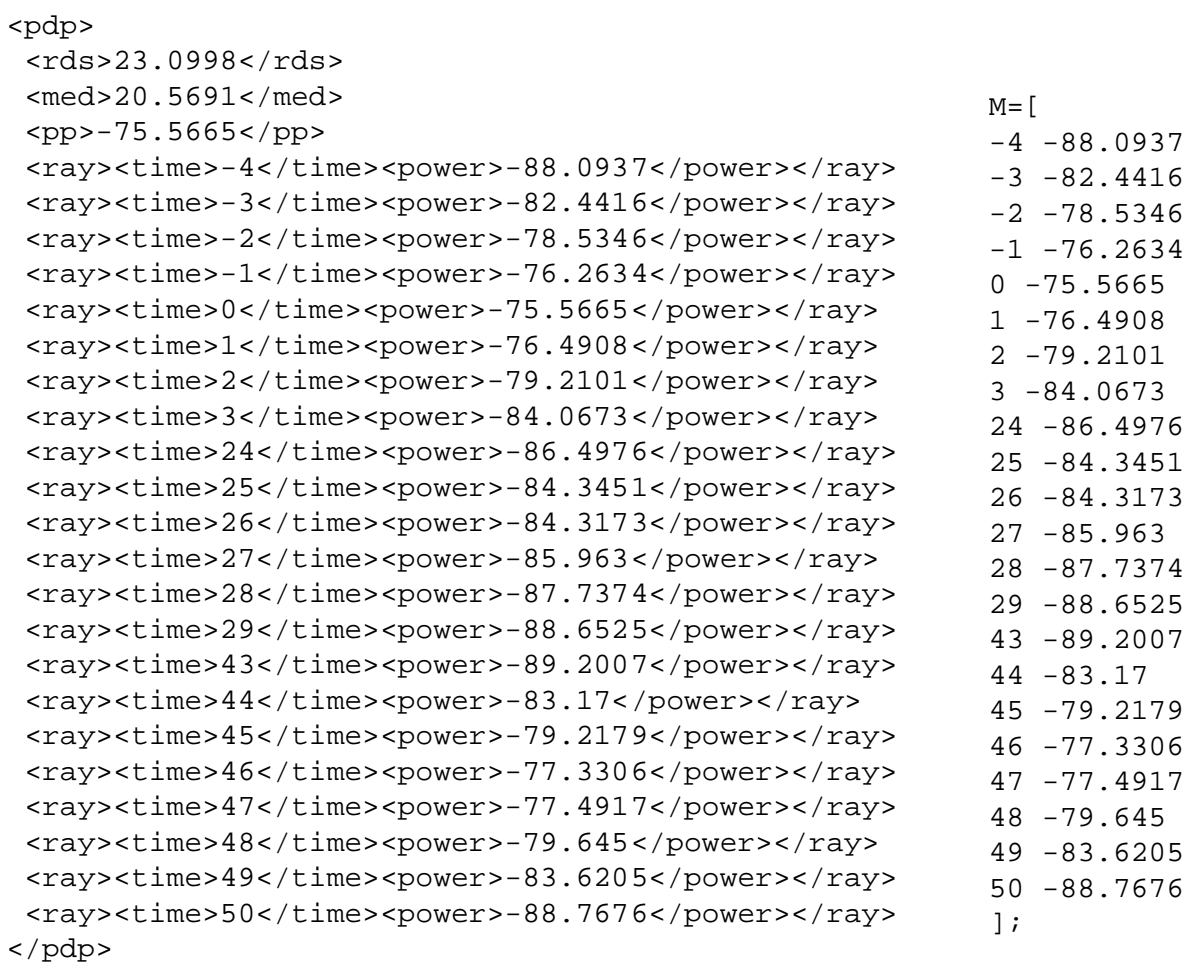

Fig. 8. (left) An example PDP in XML. The data corresponds to a simulated channel in the corridor of the fourth floor of Durham Hall, Virginia Tech. The post processor samples the channel at $1 \mathrm{~ns}$ time intervals to match the output of a channel sounder. (right) Matlab encoding of the PDP on the left, output by the parser generated from the schema in Fig. 7.

Consider a derivation of $A$ that is no longer leftrecursive (i.e., does not have a prefix of $\{d\} A$ ). All such derivations can be written as

$$
\begin{gathered}
\left.A \Rightarrow+c_{i_{1}}\right\},\left\{c_{i_{2}}\right\}, \ldots,\left\{c_{i_{k}}\right\}, \\
\beta_{j}, \alpha_{i_{k}}, \ldots, \alpha_{i_{2}}, \alpha_{i_{1}},
\end{gathered}
$$

where $\beta_{j}, 1 \leq j \leq m$, stops left recursion after (at least) $k+1$ steps and $1 \leq i_{1}, i_{2}, \ldots, i_{k} \leq n$ represent the choices for $\alpha_{i}$ in the derivation. Suppose $\beta_{j} \Rightarrow^{*} \gamma\{d\} \theta$ or $\alpha_{i} \Rightarrow^{*} \gamma\{d\} \theta$. The sequence of codes $c_{i_{1}}, c_{i_{2}}, \ldots, c_{i_{k}}$ must be executed before code $d$, but the LL(1) parser will only determine this sequence after it has parsed all of $\beta_{j}, \alpha_{i_{k}}, \ldots, \alpha_{i_{2}}, \alpha_{i_{1}}$. Thus, eliminating left recursion entails delaying user code execution in all but the trivial cases mentioned above.

Example 2 Left factoring of L-attributed definitions poses similar problems. Consider the following schema and L-attributed definition (a more realistic version of this example would have a repetition in place of the $\mathrm{x}$ element):

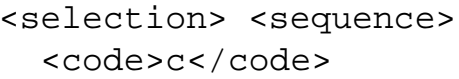

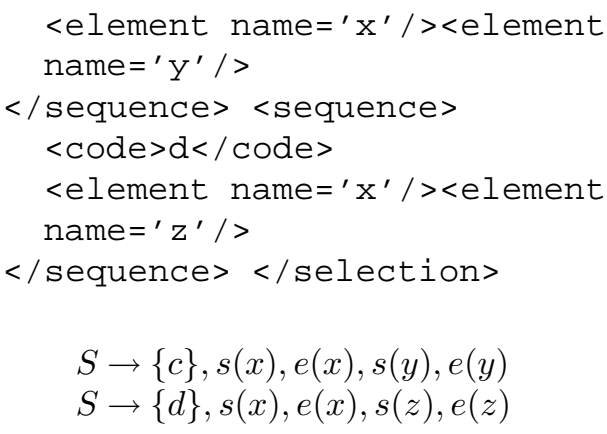

$$
S \rightarrow\{c\}, s(x), e(x), s(y), e(y)
$$$$
S \rightarrow\{d\}, s(x), e(x), s(z), e(z)
$$

The decision about whether to execute code c or d cannot be made until $s(y)$ or $s(z)$ is processed. However, removing user codes makes this L-attributed definition easy to refactor. Again, we can show a more general condition.

Observation 3 Consider a set of all productions for a non-terminal $A$ written as

$$
\begin{aligned}
A \rightarrow & \alpha_{1} \beta_{1}\left|\alpha_{2} \beta_{2}\right| \cdots \\
& \quad\left|\alpha_{n} \beta_{n}\right| \gamma_{1}\left|\gamma_{2}\right| \cdots \mid \gamma_{m},
\end{aligned}
$$

such that $\alpha_{1}^{\prime}=\alpha_{2}^{\prime}=\cdots=\alpha_{n}^{\prime}=\alpha \neq \epsilon\left(\alpha^{\prime}\right.$ denotes $\alpha$ with all user code removed) and $\alpha$ is not a prefix of any 
$\gamma_{1}^{\prime}, \gamma_{2}^{\prime}, \ldots, \gamma_{m}^{\prime}$. Let the length of $\alpha$ be maximum and the lengths of $\alpha_{i}, 1 \leq i \leq n$, be minimum subject to $n \mathfrak{e} 2$, in which case this representation of $A$ is unique. $A$ can be left-factored without delaying execution of user code if and only if

1. no rewriting of $A$ in the above form exists (no two definitions of $A$ share the same prefix, less user codes), or

2. $\alpha_{1}=\alpha_{2}=\cdots=\alpha_{n}$ ( same codes to the left) and $A \rightarrow \gamma_{1}\left|\gamma_{2}\right| \cdots \mid \gamma_{m}$ can be left-factored.

To summarize, we implement bindings in terms of Lattributed definitions from parsing theory. These bindings work well in practice, but, in theory, annotating a schema that can be rewritten in LL(1) form can make it no longer rewritable in LL(1) form. This difficulty is inherent in L-attributed definitions. We currently assume that the user is responsible for resolving such conflicts. In practice, schemas for PSE data rarely require complicated grammars. Repetitions take care of most of the recursive schema definitions. To make LL(1) parsing possible, troublesome content can be simply enclosed in an extra XML element, whose start and end tags disambiguate the transitions of the LL(1) parser.

\section{Conversion}

Conversion is the cornerstone of a system's ability to handle changes and interface mismatches. Conversion in a PSE helps to retain historical data and facilitates inclusion of new components. We use change detection principles from [11], with a few important differences. First, our goal is not merely to detect changes, but to make PSE components work despite the changes. Second, we detect changes in the schema, not in the data. The PSE environment must guarantee that the data is in the right format for the component. The job of the component is to process any data instance that conforms to the right format. Last, change detection and conversion are local to the extent possible. Locality is a virtue not only because it allows for stream processing, but also because it limits sporadic conversions between unrelated entities.

Similarly to the two previous sections, this section starts with a comprehensive example. Then, we describe the core of the conversion algorithm and outline its limitations. Finally, we extend the initial algorithm to handle content replacements: unit conversion and user-defined conversion filters. At this point, it should not come as a surprise to the reader that most of the technical limitations of conversion are due to binding codes, not to the nature of the schema language. Therefore, the tedious details of handling binding codes are omitted. The emphasis is on non-technical limitations. What forms of semantic conversions can be 'syntactized' in a schema language? When does such 'syntactization' back fire and produce undesired outcomes?

The functional statement of the conversion problem can be given as follows. Given the actual schema $S_{a}$ and the required schema $S_{r}$, replace binding codes in $S_{a}$ with binding codes in $S_{r}$ and conversion codes to obtain the conversion schema $S_{c}$. $S_{c}$ must describe precisely the documents described by $S_{a}$, but perform the same bindings as $S_{r}$.

Example 3 Figure 9 depicts two slightly different schemas for antenna descriptions in $S^{4} W$. The schema at the bottom (actual schema) was our first attempt at defining a data format for antenna descriptions. This version supported only one antenna type and exhibited several inadequate representation choices. E.g., polar coordinates should have been used instead of Cartesian coordinates because antenna designers prefer to work in the polar coordinate system. Antenna gain was not considered in the first version because its effect is the same as that of changing transmitter power. However, this seemingly unnecessary parameter should have been included because it results in a more direct correspondence of simulation input to a physical system.

The schema at the top of Fig. 9 (required schema) improves upon the actual schema in several ways. It better adheres to common practices and supports more antenna types. However, this schema is different from the actual schema, while compatibility with old data needs to be retained (requirement 2). Figure 10 illustrates how addition of conversion and binding codes to the actual schema solves the compatibility problem. A parser generated from the conversion schema in Fig. 10 will recognize the actual data and provide the required binding.

Following [11], the basic assumption of the conversion algorithm is that the actual schema $S_{a}$ can be converted to the required schema $S_{r}$ by some sequence of 'standard' edits. This sequence of edits is called an edit script. Once the possible types of edits are defined (what we can call a 'conversion library'), the job of the conversion algorithm is to (a) find an edit script that transforms the actual schema $S_{a}$ to the required schema $S_{r}$ and (b) express this edit script as data transformations, not schema transformations. In other words, the 


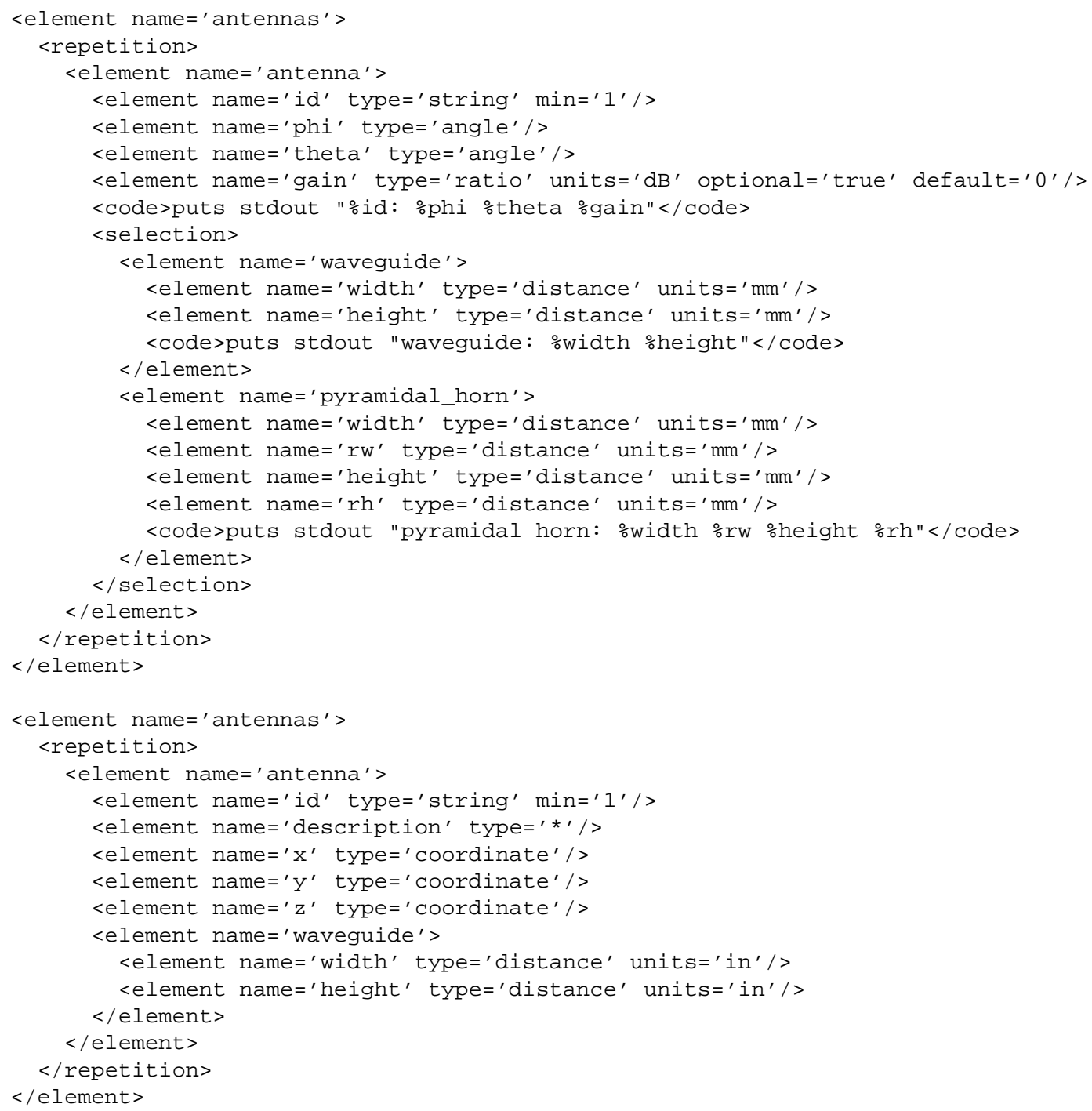

Fig. 9. Two slightly different schemas for a collection of antennas. The component requires the top schema, but the data conforms to the bottom schema. The bottom schema (a) represents antenna orientation in Cartesian coordinates, not polar coordinates, (b) lacks antenna gain, (c) requires antenna descriptions, (d) measures antenna dimensions in inches, not millimeters, and (e) covers only one antenna type. The schema at the bottom does not contain binding codes because they are irrelevant for this example. All binding codes are in Tcl.

conversion algorithm looks for a systematic procedure that converts actual data instances that conform to $S_{a}$ to the required format $S_{r}$. This procedure is expressed as a conversion schema $S_{c}$ that has the structure of $S_{a}$, but binding codes from $S_{r}$ and the conversion library. $S_{c}$ is then used to generate a parser that parses data instances conforming to $S_{a}$ and acts as if it parsed data instances conforming to $S_{r}$.

Our conversion algorithm supports four kinds of schema edits:

1. generalization,

2. restriction,
3. reordering, and

4. replacement.

We use these terms in reference to the required schema, e.g., 'the required schema is a generalization of the actual schema.' Generalization and restriction of schema trees are similar to insertions and deletions in sequence alignment problems. Reordering and replacement mostly retain their standard meaning, except we consider replacements of sets of schema blocks, not individual schema blocks. We first reduce the problem of converting trees to an easier problem of converting sequences (see Fig. 11). Sequence conversion (rule $Q$ ) 


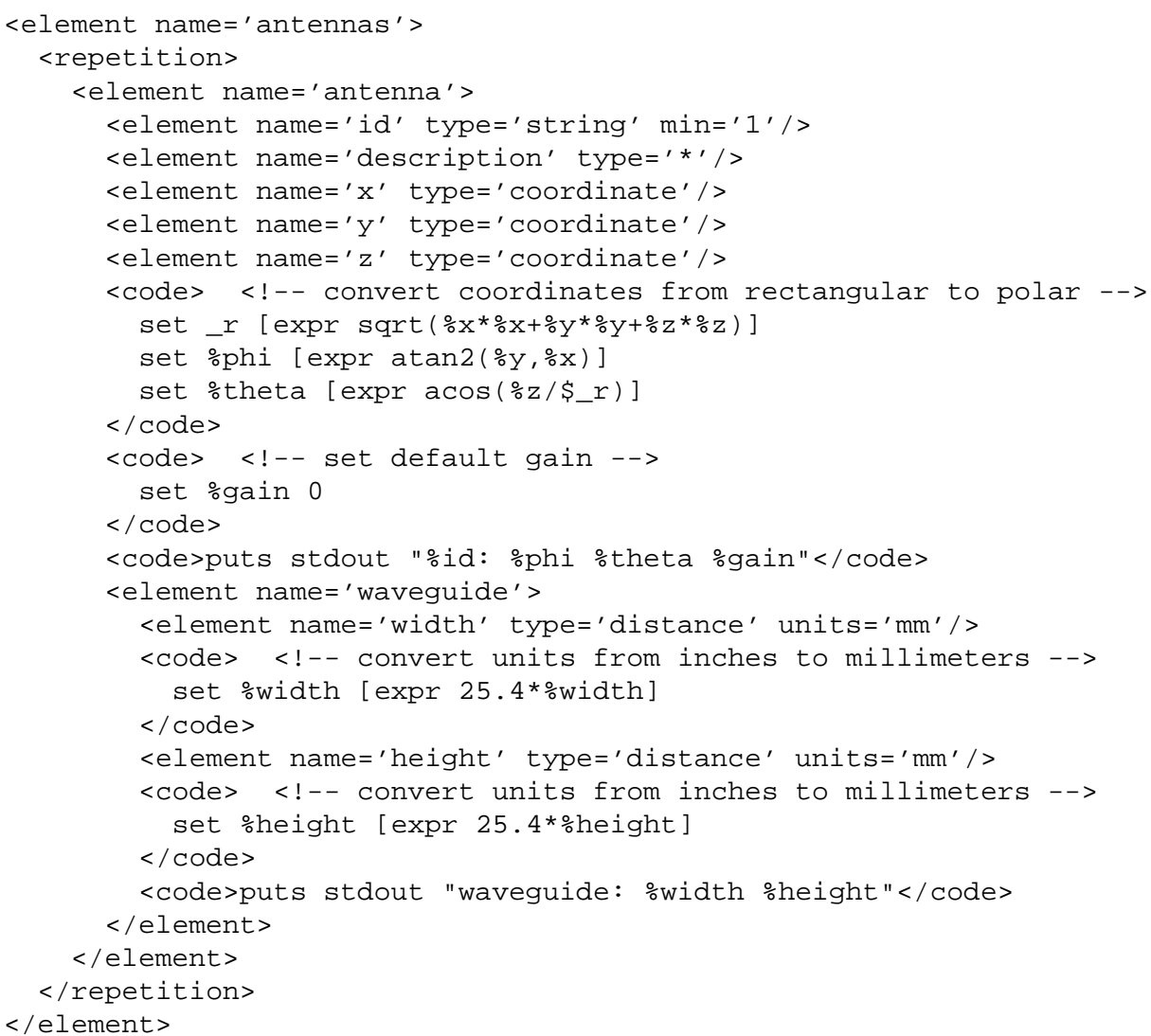

Fig. 10. Actual schema from Fig. 9 (bottom) after inserting conversion and binding codes. This schema describes the actual documents, but provides the bindings of the required schema (top of Fig. 9). We use $\_r$ instead of $\% r$ because the latter could interfere with another use of the name $r$.

in this initial formulation performs all conversions but replacements. Then, we slightly restrict this definition to make it practical and generalize rule $Q$ to accommodate replacements (unit conversion and user-defined conversion filters).

The conversion algorithm revolves around the 'determines' relation between schemas. Intuitively, an actual schema $S_{a}$ should determine a required schema $S_{r}$ if any document that conforms to $S_{a}$ contains sufficient information to construct an 'appropriate' document that conforms to $S_{r}$. 'Appropriate' here is obviously a domain-specific notion, and in the absence of a domain theory, there is no hard and fast measure of 'appropriateness'. Given two slightly different schemas, only a domain expert can tell whether or not it is meaningful to attempt a conversion from one form to another. Therefore, our conversion rules should be viewed as heuristics that we have found to be useful enough to be supported in a conversion library. They are neither sound nor complete in an algorithmic sense (because we do not have an objective, external, mea- sure of 'conversion correctness'). Instead, they represent a tradeoff between soundness and completeness and should be carefully evaluated for use in a particular domain. With this disclaimer in mind, version 1 of the determines relation between $S_{a}$ and $S_{r}\left(S_{a}\right.$ determines $\left.S_{r} ; S_{a} \succeq S_{r}\right)$ is defined in Fig. 11. We will also find the notion of schema equivalence useful: we say that two schemas $S_{a}$ and $S_{r}$ are equivalent if $S_{a} \succeq S_{r}$ and $S_{r} \succeq S_{a}$.

The first rule $\left(D_{r}\right)$ in Fig. 11, for instance, says that a value of primitive type ('data') can be substituted for another if they have the same base type, their ranges are compatible, and they have the same units. It ensures that all primitive type constraints of $S_{r}$ are met by $S_{a}$ (restriction). Thus, $D_{r}$ is simply a definition of type derivation by range restriction (the ' $r$ ' subscript in this and other rules stands for restriction; similarly, the 'g' subscript stands for generalization). Rules $E, P$, and $R$ state the obvious: two black boxes are compatible if they have compatible wrappers (restriction) and compatible contents (any conversions). Rule $C$ says 


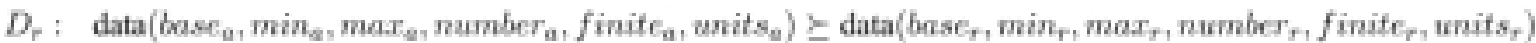

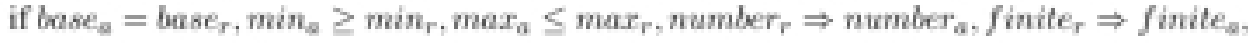

units $s_{\mathrm{a}}=$ units $_{\mathrm{r}}$

$E: \quad$ element $\left(\right.$ id $_{a}$, opt $_{\alpha}$, name $\left._{a}, C_{\alpha 1}, C_{a 2}, \ldots, C_{a n}\right) \succeq \operatorname{element}\left(i d_{r}, o p t_{r}\right.$, name $\left._{r}, C_{r 1}, C_{r 2}, \ldots, C_{r m}\right)$

if name $_{\mathrm{a}}=$ name $_{\mathrm{r}}$, opt $\mathrm{a} \Rightarrow o p \mathrm{t}_{\mathrm{r}}, Q_{\mathrm{a}}\left(C_{\mathrm{a} 1}, C_{\mathrm{a} 2}, \ldots, C_{\mathrm{ax}}\right) \succeq Q_{\mathrm{r}}\left(C_{\mathrm{r} 1}, C_{\mathrm{r} 2}, \ldots, C_{\mathrm{rm}}\right)$

$E_{g}: X_{a}\left(i d_{s}, o p t_{a}, \ldots\right) \succeq \operatorname{element}\left(i d_{r}, o p t_{r}\right.$, name $\left._{r}, C_{r 1}, C_{r 2}, \ldots, C_{r m}\right)$

if $o p t_{a} \Rightarrow o p t_{r}, Q_{a}\left(X_{a}\left(i d_{a}, o p t_{a}, \ldots\right)\right) \succeq Q_{r}\left(C_{r 1}, C_{r 2}, \ldots, C_{r m}\right)$

$E_{r}$ : element $\left(i d_{\alpha}\right.$, opt $_{\alpha}$, name $\left._{\alpha}, C_{\alpha 1}, C_{\alpha 2}, \ldots, C_{\mathrm{an}}\right) \succeq X_{\mathrm{r}}\left(\mathrm{id}_{r}\right.$, opt $\left.t_{r}, \ldots\right)$

if opt $t_{a} \Rightarrow o p t_{r}, Q_{a}\left(C_{a 1}, C_{a 2}, \ldots, C_{a n}\right) \succeq X_{r}\left(i d_{r}, o p t_{r}, \ldots\right)$

$P: \quad$ sequence $\left(i d_{0}, o p t_{0}, C_{a 1}, C_{a 2}, \ldots, C_{a n}\right) \succeq$ sequence $\left(i d_{p}, o p t_{p}, C_{r 1}, C_{r 2}, \ldots, C_{r m}\right)$

if opt $t_{n} \Rightarrow o p t_{r}, Q_{a}\left(C_{a 1}, C_{a 2}, \ldots, C_{a n}\right) \succeq Q_{r}\left(C_{r 1}, C_{r 2}, \ldots, C_{r m}\right)$

$P_{g}: X_{\alpha}\left(\right.$ id $_{\alpha}$, opt $\left._{a}, \ldots\right) \succeq \operatorname{sequence}\left(i d_{r}\right.$, opt $\left._{r}, C_{r 1}, C_{r_{2}}, \ldots, C_{r m}\right)$

if opt $t_{a} \Rightarrow o p t_{r}, Q_{a}\left(X_{a}\left(i d_{a}, o p t_{a}, \ldots\right)\right) \succeq Q_{r}\left(C_{r 1}, C_{r 2}, \ldots, C_{r m}\right)$

$P_{r}:$ sequence $\left(i d_{a}, o p t_{a}, C_{a 1}, C_{a 2}, \ldots, C_{a n}\right) \geq X_{r}\left(i d_{r}, o p t_{r}, \ldots\right)$

if opt $t_{a} \Rightarrow o p t_{r}, Q_{a}\left(C_{a 1}, C_{a 2}, \ldots, C_{a n}\right) \succeq X_{r}\left(i d d_{r}, o p t_{r}, \ldots\right)$

$C: \quad$ selection $\left(i d_{\mathrm{a}}, o p t_{\mathrm{a}}, C_{\mathrm{al}}, C_{\mathrm{a} 2}, \ldots, C_{\mathrm{an}}\right) \succeq \operatorname{selection}\left(i_{\mathrm{r}}, o p t_{\mathrm{r}}, C_{\mathrm{r} 1}, C_{\mathrm{r} 2}, \ldots, C_{\mathrm{rm}}\right)$

if opt $t_{a} \Rightarrow o p t_{r}, \forall C_{a i}:\left(\exists ! C_{r j}: C_{a i} \succeq C_{r j}\right)$

$C_{g}: X_{\alpha}\left(\right.$ id $\left._{\alpha}, o p t_{a}, \ldots\right) \succeq \operatorname{selection}\left(i d_{r}, o p t_{r}, C_{r 1}, C_{r 2}, \ldots, C_{r m}\right)$

if opt $t_{\alpha} \Rightarrow o p t_{r},\left(\exists ! C_{r j}: X_{a}\left(i d_{s}, o p t_{a}, \ldots\right) \succeq C_{r j}\right)$

$R$ : repetition $\left(i d_{\alpha}, o p t_{a}, \min _{\alpha}, \max _{\alpha}, C_{e 1}, C_{e 2}, \ldots, C_{\mathrm{ax}}\right) \geq \operatorname{repetition}\left(i d_{r}, o p t_{r}, \min _{r}, \max _{r}, C_{\mathrm{rl}}, C_{r 2}, \ldots, C_{r m}\right)$ if $\min _{0} \geq \min _{r}, \max _{\alpha} \leq \max _{r}, o p t_{0} \Rightarrow o p t_{r}, Q_{0}\left(C_{a 1}, C_{a 2}, \ldots, C_{a x}\right) \succeq Q_{r}\left(C_{r 1}, C_{r 2}, \ldots, C_{r m}\right)$

$R_{g}: X_{s}\left(\right.$ id $\left._{a}, o p t_{a}, \ldots\right) \succeq$ repetition $\left(i d_{r}, o p t_{r}, \min _{r}, \max _{r}, C_{r 1}, C_{r 2}, \ldots, C_{r m}\right)$

if $\min _{r} \leq 1, \max _{r} \geq 1, o p t_{0} \Rightarrow o p t_{r}, Q_{0}\left(X_{0}\left(i d_{0}, o p t_{0}, \ldots\right)\right) \succeq Q_{r}\left(C_{r 1}, C_{r 2}, \ldots, C_{r m}\right)$

$F: \quad \operatorname{ref}\left(i d_{\mathrm{a}}\right) \succeq \operatorname{ref}\left(i d_{\mathrm{r}}\right)$

if $X_{o}\left(i d_{s}, o p t_{0}, \ldots\right) \succeq X_{r}\left(i d_{r}, o p t_{p}, \ldots\right)$

$Q: \quad Q_{a}\left(C_{a 1}, C_{a 2}, \ldots, C_{a n}\right) \succeq Q_{r}\left(C_{r 1}, C_{r 2}, \ldots, C_{r m}\right)$

if $v C_{r j}\left(\ldots, o p t_{r j}, \ldots\right):\left[\left(\exists ! C_{a i}: C_{a i} \succeq C_{r j}\right)\right.$ or $\left.\left(o p t_{r j}\right)\right]$

Fig. 11. Version 1 of the 'determines' relation $X_{a}\left(i d_{a}, o p t_{a}, \ldots\right) \succeq X_{r}\left(i d_{r}, o p t_{r}, \ldots\right)$ between an actual schema block $X_{a}\left(i d_{a}, o p t_{a}, \ldots\right)$ and a required schema block $X_{r}\left(i d_{r}, o p t_{r}, \ldots\right)$. We use the non-XML notation from Fig. 4 plus $X_{a}\left(i d_{a}, o p t_{a}, \ldots\right)$ and $X_{r}\left(i d_{r}, o p t_{r}, \ldots\right)$ are shortcuts for any schema block (data blocks are never optional and have empty ids). $\Rightarrow$ means logical implication and $\exists$ ! means 'there exists a unique.' The rules are applied top to bottom, left to right. The first matching rule wins (no backtracking). This definition will be later restricted to make it computable and rule $Q$ will be extended to handle replacements.

that any choice in $S_{a}$ must uniquely determine some choice in $S_{r}$ (restriction). Rule $Q$ enforces that every block in $S_{r}$ is uniquely determined by some block in $S_{a}$. This formulation of rule $Q$ ignores extra blocks in $S_{a}$ (restriction), permits optional elements in $S_{r}$ to be unmatched (generalization), and allows for contents reordering. Rule $F$ deals with references. Only rules $D_{r}$,
$E, P, C$, and $R$ are sound. Rule $F$ looks sound, but it makes the determines relation not computable. Rule $Q$ is unsound primarily because it ignores 'unnecessary' blocks in $S_{a}$.

Rules $E_{g}, P_{g}, C_{g}$, and $R_{g}$ handle generalizations across schema blocks of (possibly) different types. Their counterparts $E_{r}$ and $P_{r}$ handle symmetric re- 
strictions (why is there no $C_{r}$ or $R_{r}$ ?). Rule $C_{g}$ was demonstrated in the example above. It is a base case for rule $C$. Rule $C_{g}$ states that one way to generalize a schema block is to enclose it in a selection, i.e., provide more choices in $S_{r}$ than were available in $S_{a}$. This rule is sound. Rules $E_{g}, P_{g}$, and $R_{g}$ have similar motivations, but they are unsound. Essentially, we assume that decorating any black box with any number of wrappers does not change the meaning of the black box (generalization). Similarly, we assume that wrappers can be freely removed to expose the black box (restriction).

Consider a sequence of schemas that describes some physical system in progressively greater detail. Suppose some subsystem is described by a single parameter. Common practice is to allocate a single schema block to this subsystem. What happens when a more detailed description of this subsystem is incorporated into the schema? Chances are, the original schema block allocated to the subsystem will be either (a) augmented with more contents (restriction part of rule $Q$ ) or (b) wrapped in another block. The generalization and restriction rules handle case (b). However, blind application of these rules can lead to disaster because these rules disregard some semantic information. Examples will make these points clearer.

Example 4 One common trick used to improve wireless system performance is space-time transmit diversity (STTD). Instead of a single transmitter antenna, the base station uses two transmitter antennas separated by a small distance. PDPs are very sensitive to device positioning, so two uncorrelated transmitter antennas can produce widely different signals at the same receiver location. If the signal from one of the antennas is weak, the signal from another antenna will probably be strong, so the overall performance is expected to improve. Consider how addition of STTD to the ray tracer affects the schema of the transmitter file. The original schema is on the left and the new schema (with STTD support) is on the right. The second antenna is optional because STTD is not used in every system due to cost considerations.

(continued on next page)

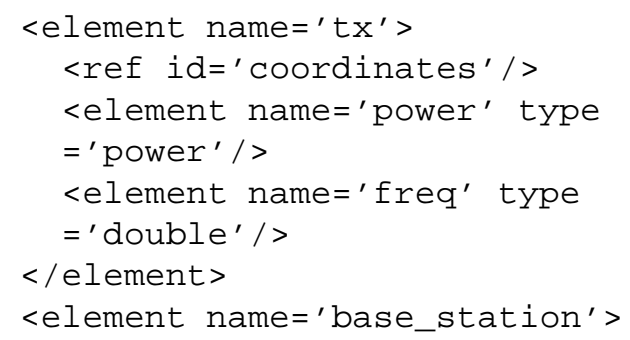

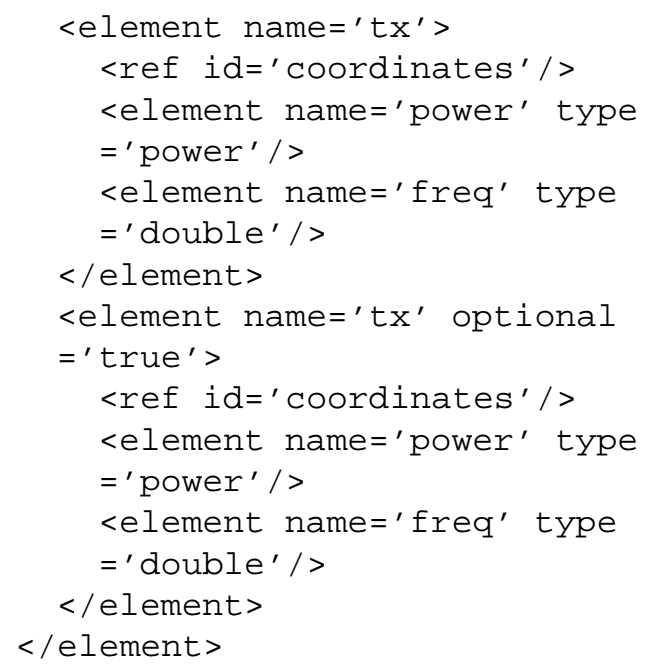

The new ray tracer should be able to work with old data because it supports one or two transmitter antennas. The old ray tracer should be able to work with new data, albeit the results will be approximate when the new data contains two transmitter antennas. Further generalizing this example to $n$ transmitter antennas would require a repetition. We support conversion to repetitions, but not from repetitions. For this example, we could extract any antenna because they usually have the same parameters and are positioned close together. However, we cannot extract an arbitrary ray from a PDP because the ray with maximum power is usually intended. Extracting any other ray would typically produce nonsense results.

Example 5 Havoc can result if rules $E_{r}$ and $E_{g}$ are applied to the same element. Element names have semantic meaning, but this particular composition of rules allows arbitrary renaming of elements. Such renaming would make the following two schemas equivalent.

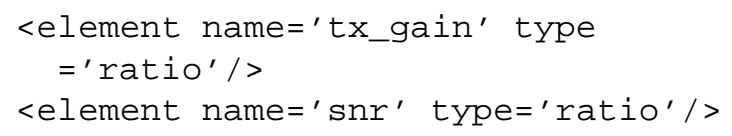

Even though both transmitter antenna gain and signal-to-noise ratio are ratios measured in the same units $(d B)$, they convey largely different information. We avoid such blatant mistakes by limiting the application of generalization and restriction rules. In particular, no element can be renamed.

As the last example illustrates, the 'determines' relation in Fig. 11 needs to be restricted. It is helpful to redefine this relation in terms of a context-free grammar that describes $S_{a} S_{r}$. Let the terminals be element(, 
sequence(, selection(, repetition(, ref(, data(, ), and all element names and other values used in two schemas under consideration. Let the nonterminals be the labels of the rules in Fig. 11, a special start non-terminal $A$, and intermediate non-terminals introduced by the rules. We can formally define the necessary restrictions by limiting the shape of the parse tree for $S_{a} S_{r}$. Consider a path $R_{1}, R_{2}, \ldots, R_{n}, n>0$, from some internal node $R_{1} \neq A$ to some internal node $R_{n} \neq A$, where all $R_{i}, 1 \leq i \leq n$, are rule labels. If $\mathrm{R}$ is the set of restriction rules and $\mathrm{G}$ is the set of generalization rules, we require that $\left(R_{i} \in \mathrm{R}\right)$ implies $\left(R_{i-1} \notin \mathrm{G}\right.$ and $\left.R_{i+1} \notin \mathrm{G}\right)$, i.e., restriction and generalization rules cannot be applied in sequence. This restriction of the parse tree disallows renaming of elements, but does not limit the number of wrappers around black boxes. Bounded determination deals with the latter problem. We say that $S_{a} k$-determines $S_{r}\left(S_{a} \succeq^{k} S_{r}\right)$ if no path $R_{1}, R_{2}, \ldots, R_{n}$ contains a substring of (possibly different) generalization (restriction) rules of length greater than $k$. We leave it up to the reader to appropriately restrict rule $F$ (reference). These restrictions make the 'determines' relation computable and enforce locality of conversions. As a side effect, we have shown that the problem of constructing a conversion schema $S_{c}$ from the actual schema $S_{a}$ and the required schema $S_{r}$ can be reduced to validation and binding (parsing and translation). However, schema conversion need not work with streams of data, so a parser more powerful than a predictive parser should be used.

It remains to consider requirements 4 and 5: unit conversion and user-defined conversion filters (replacements). Let $D$ be a set of all primitive types derived from double (recall that a primitive type is defined by the base type, the range of legal values, and a unit expression). Unit conversion, e.g., converting $\mathrm{kg} / \mathrm{m}^{2}$ to $\mathrm{lb} / \mathrm{in}^{2}$, is the simpler of the two replacements. Both actual and required unit expressions are converted to a canonical form (e.g., a fraction of products of sums of $\mathrm{CI}$ units or $\mathrm{dB}$ ) and then the conversion function is found. Unit conversions are functions of the form

$$
U: D_{a} \rightarrow D_{r},
$$

where $D_{a}, D_{r} \in D$ are specific primitive types. Userdefined conversion filters are functions of the form

$$
\begin{aligned}
& H: D_{a 1} \times D_{a 2} \times \cdots \times D_{a n} \\
\rightarrow & D_{r 1} \times D_{r 2} \times \cdots \times D_{r m},
\end{aligned}
$$

where $n, m>0$ and all $D_{a i}, D_{r j} \in D, 1 \leq$ $i \leq n, 1 \leq j \leq m$, are specific primitive types.
Arithmetic operators and common mathematical functions are allowed in user-defined conversion filters. Each user-defined conversion filter is tagged with element names name $_{a 1}$, name $_{a 2}, \ldots$, name $_{a n}$ and name $_{r 1}$, name $_{r 2}, \ldots$, name $_{r m}$ that determine when the filter applies. Such filters define rules of the form

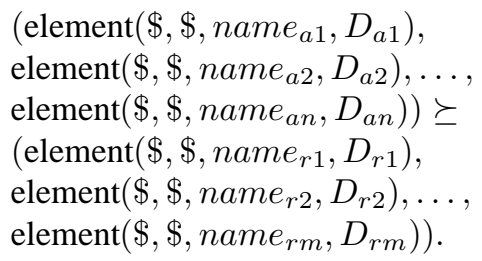

Both kinds of filters are compiled into codes such as shown in Fig. 10. Rule $Q$ is modified to take advantage of replacements. Basically, we are looking for (unique) partitions of the actual schema blocks $C_{a 1}, C_{a 2}, \ldots, C_{a n}$ and required schema blocks $C_{r 1}, C_{r 2}, \ldots, C_{r m}$ such that each set of schema blocks in the required partition is determined by some set of schema blocks in the actual partition. Determination can proceed through the rules in Fig. 11, unit conversions, and user-defined conversion filters (if everything else fails, optional blocks in the required schema can remain unmatched).

The ultimate goal of the conversion algorithm is to find a meaningful edit script. However, this goal is impossible to achieve without knowledge of the domain. What happens when several edit scripts exist, i.e., the problem of finding an edit script is ambiguous? Depending on the nature of the ambiguity, we can choose any edit script, the minimal (in some sense) edit script, or to refuse to perform conversion. The conversion algorithm described here either settles for some local minimum (e.g., rule $E$ is preferred over rule $E_{g}$ ) or requires uniqueness of conversions (rules $C, C_{g}$, and most of rule $Q$ ). Ambiguity remains an open problem that is unlikely to be solved by a syntactic conversion algorithm. Following the principle of least user astonishment, we choose to reject most of ambiguous conversions.

Finally, let us consider how binding codes limit conversion. We omit formal treatment of the problem and limit the discussion to an example. It is easy to see that conversion may require delaying binding code execution. This should not be surprising since one kind of conversion is reordering.

Example 6 Consider a required schema with binding codes (left) and an actual schema (right). 


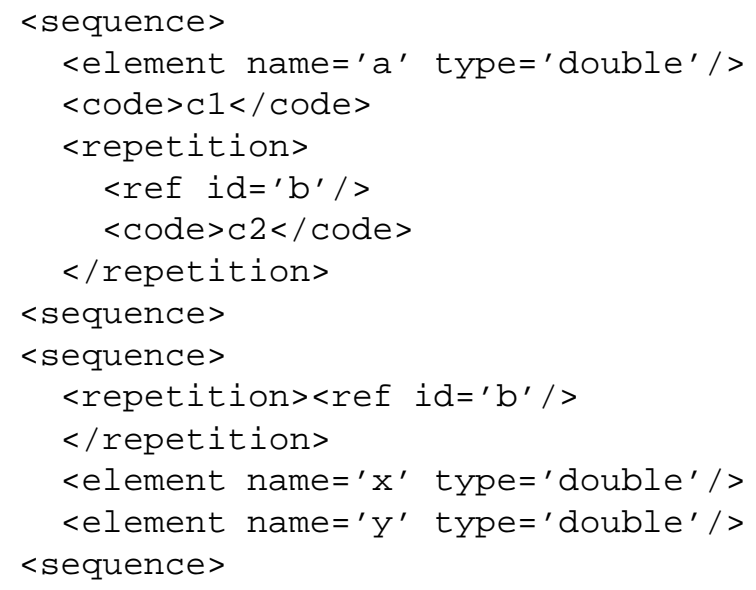

Assume that there exists a user-defined conversion filter that calculates a from $\mathrm{x}$ and $\mathrm{y}$. If we ignore binding code $\mathrm{c} 2$, conversion is clearly local. However, conversion with $\mathrm{C} 2$ present will require delaying all executions of $\mathrm{C} 2$ until $\mathrm{C} 1$ is executed. The latter can only happen when the last piece of the schema is matched. In other words, binding codes should be placed as late as possible in the schema.

This section presented a number of local conversions appropriate for PSE data. Conversions are carried out by extra codes injected in the actual schema. The conversion algorithm was built around the 'determines' relation between schemas. The algorithm has some technical limitations related to binding codes, but its major limitation is conceptual. Conversion, in the form presented here, is syntactic. It is based on the weak semistructured data model, not on the underlying domain theory (wireless communications). Therefore, we can only speculate about the causes of differences between the actual and required schemas. There is no guarantee that automatic conversion will produce meaningful results. A stronger data model is necessary to perform complex, yet meaningful, conversions.

\section{Integration with a PSE}

A complete PSE requires functionality far beyond validation, binding, and conversion. BSML ensures that the components can read streams of XML data, but it does not support tasks such as scheduling, communication, database storage and retrieval, connecting multiple components into a given topology, and computational steering. We broadly call software that performs all of these tasks an execution manager. Figure 12 illus- trates how BSML software and the execution manager function together.

From a systems point of view, BSML schemas are metadata and the BSML software is a parser generator. Recall that the parser generator generates parsers that perform validation, binding, and conversion functions (every such generated parser will be able to take input data and stream it through the component). Both the data and the metadata are stored in a database. We can distinguish three kinds of metadata: schemas, component metadata, and model instance metadata. Only one form of metadata (schemas) was described in this paper. Component metadata contains component's local parameters, such as executable name, programming language, and input/output port schemas. It is the kind of metadata used in CCAT. Model instance metadata, i.e., component topology and other global execution parameters, serves a purpose similar to GALE's workflow specifications. It supports our requirement 3.

A parser is lazily generated for each used combination of component's input port schema (required schema) and the schema of the data instance connected to this port (actual schema). Component metadata specifies how linking must be performed (e.g., which of the three kinds of bindings to use). Component instances are further managed by the execution manager. Model instance metadata specifies how to execute the model instance (e.g., the topology and the number of processors), while model instance data serves as the actual (data) input to the model instance. To summarize, the BSML parser generator creates component instances programs that take a number of XML streams as inputs and produce a number of XML streams as outputs. This representation is appropriate for management of a PSE execution environment.

\subsection{Status of prototype}

In $S^{4} \mathrm{~W}$, the execution manager is implemented in $\mathrm{Tcl} / \mathrm{Tk}$ and most of the component metadata is hardcoded. Model instance metadata consists primarily of the number of processors and a cross-product of references to model instance data. An (incomplete) example of such a specification is

'compute power coverage maps for these three transmitter locations in Torgersen Hall and show a graph of BERs with the signal-to-noise ratio varying from zero to twenty $\mathrm{dB}$ in steps of two $\mathrm{dB}$; use thirty nodes of a 200-node Beowulf cluster.' 


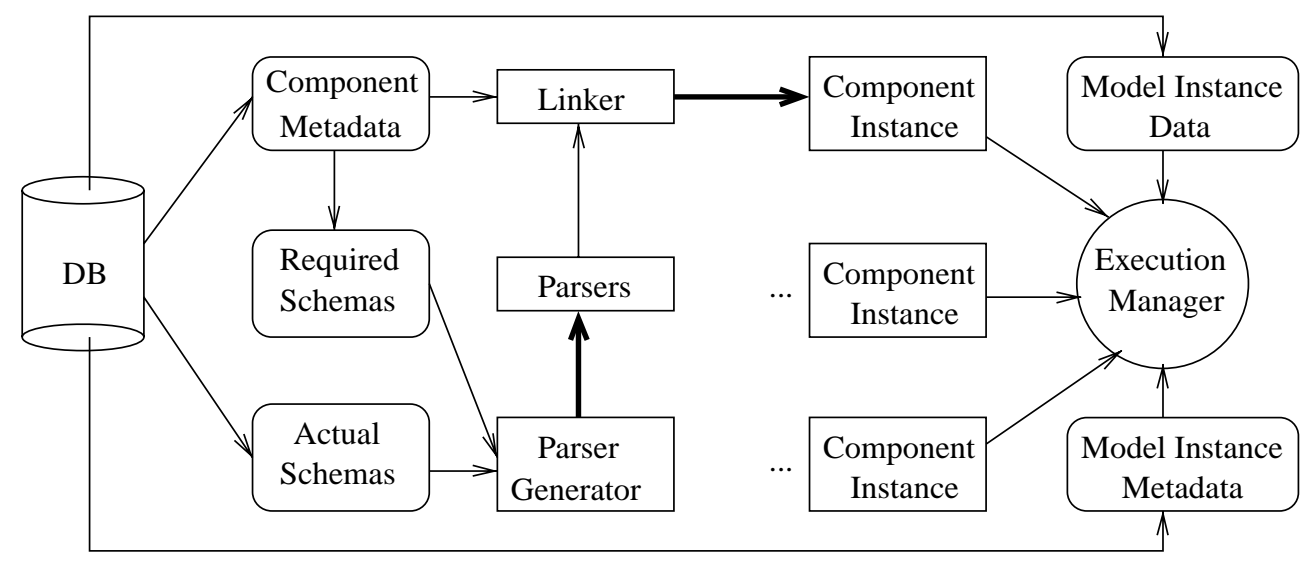

Fig. 12. BSML integration with PSE execution environment. The BSML parser generator creates parsers that handle input ports of each component. Execution manager controls the execution of a model instance that consists of components, model instance data, and model instance metadata. Figure 1 partially defines one such instance.

PostgreSQL and the filesystem serve the role of the database. Large files (e.g., floor plans) are typically stored in the filesystem and small ones (e.g., PDPs) are usually imported into PostgreSQL. The parser generator is written in SWI Prolog. It generates parsers in Tcl. Our choice of languages was driven by the existing in-house computational environment and the ease of prototyping in these languages; their selection is not the result of a systematic investigation of implementation options. Currently, the generated parsers are used mostly in the execution manager, visualization components, and database interfacing components.

\section{Discussion}

We have described the use of validation, binding, and conversion facilities to solve data interchange problems in a PSE. Since all three concepts are closely related to parsing and translation, viewing application composition in terms of data management uncovers wellunderstood solutions to interface mismatch problems. The semistructured data model allows us to syntactically define several forms of conversions that are usually implemented by hand-written mediators in PSEs. Such automation reduces the cost of PSE development and, more importantly, brings PSEs closer to their ultimate goal - namely, PSE users should be solving their domain-specific problems, not be beset by the technical details of component composition in a heterogeneous computing environment.

Several extensions to the present work are envisioned. First, the expressiveness of schema languages for data interchange and application composition can be formally characterized. This will allow us to reason about requirements such as stream processing from a modeling perspective. Such a study will also lead to a better understanding of the roles that a markup language can play in a PSE. Second, dataflow relationships between components can be made explicit. BSML guarantees that any component instance be able to process streams of data, but synchronization issues are meant to be resolved by the execution manager. Tighter integration of BSML and composition frameworks can be explored. Finally, the overall view of a PSE as a semistructured data management system deserves further exploration. For example, it seems possible to automatically generate workflow specifications from queries on a semistructured database of simulation results.

Any good problem solving facility is characterized by 'what it lets you get away with.' BSML is unique among PSE projects in that it allows a modeler or engineer to flexibly incorporate application-specific considerations for data interchange, without insisting on an implementation vocabulary for components. 
A BSML DTD

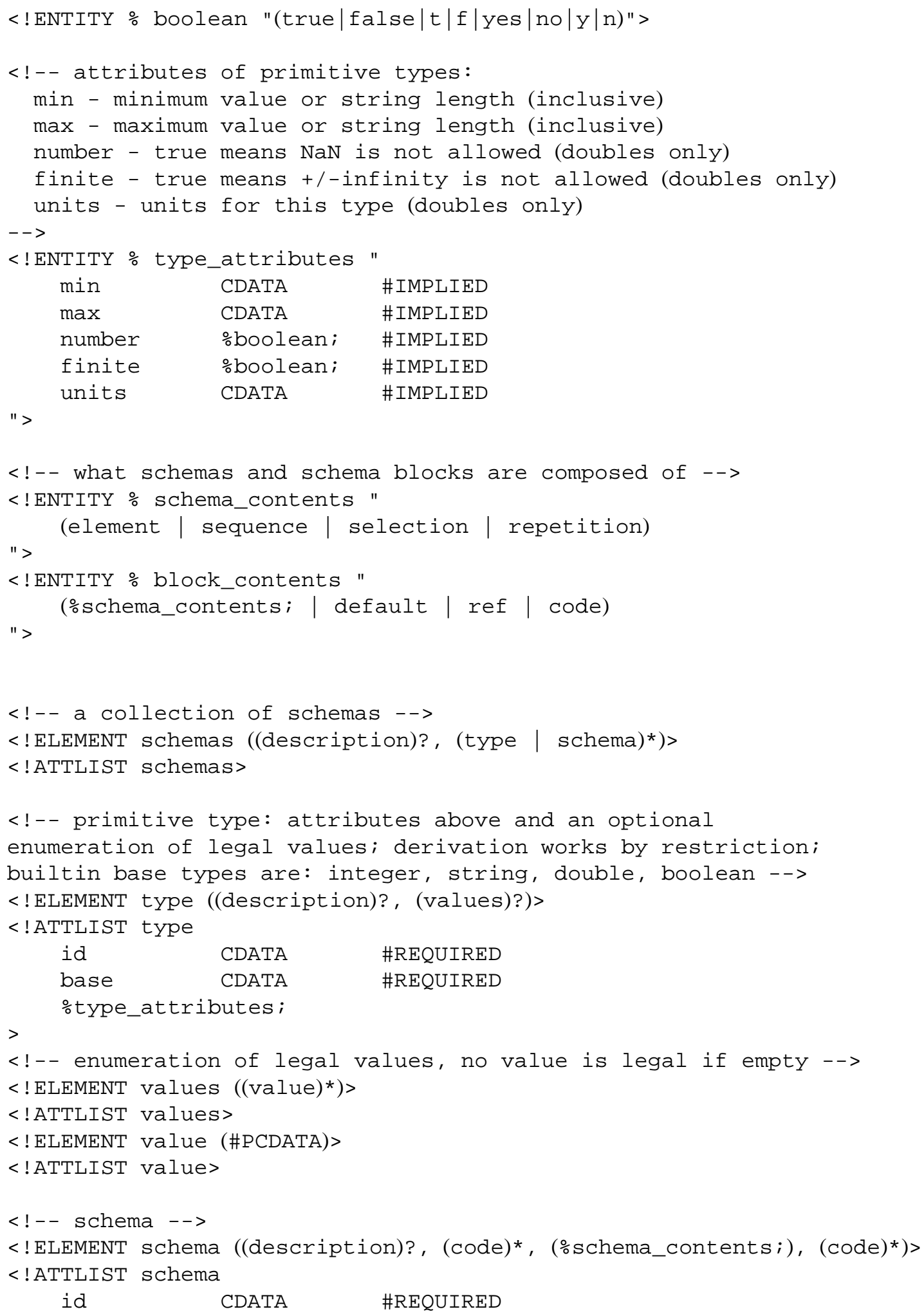


$>$

<!-- an element can contain either

(a) character data of a primitive type (type attribute is present),

(b) zero or more schema blocks (type attribute is absent), or

(c) when type='*', any contents.

$<$ !ELEMENT element ((description)?, (attribute)*,

$\left.\left.((\text { values }) \text { ? | (ololock_contents; })^{\star}\right)\right)>$

$<$ !ATTLIST element

$\begin{array}{lll}\text { name } & \text { CDATA } & \text { \#REQUIRED } \\ \text { id } & \text { CDATA } & \text { \#IMPLIED } \\ \text { optional } & \text { oboolean; } & \text { "false" } \\ \text { type } & \text { CDATA } & \text { \#IMPLIED } \\ \text { otype_attributes; } & \\ \text { default } & \text { CDATA } & \text { \#IMPLIED }\end{array}$

$>$

<!-- an attribute must contain a value of some primitive type -->

$<$ !ELEMENT attribute ((description)?, (values)?)>

$<$ !ATTLIST attribute

$\begin{array}{lll}\text { name } & \text { CDATA } & \text { \#REQUIRED } \\ \text { id } & \text { CDATA } & \text { \#IMPLIED } \\ \text { type } & \text { CDATA } & \text { "string" }\end{array}$

otype_attributes;

default CDATA \#IMPLIED

$<$ !-- a sequence is just a grouping, for convenience -->

$<$ !ELEMENT sequence ((description)?, (\%block_contents; $\left.)^{\star}\right)>$

$<$ !ATTLIST sequence

id CDATA \#IMPLIED

optional oboolean; "false"

$>$

<!-- a selection denotes a mutually exclusive choice of contents -->

$<$ !ELEMENT selection ((description)?, (\%block_contents;)+)>

$<$ !ATTLIST selection

$\begin{array}{lll}\text { id } & \text { CDATA } & \text { \#IMPLIED } \\ \text { optional } & \text { oboolean; } & \text { "false" }\end{array}$

$>$

$<$ !-- a repetition denotes [min..max] repetitions of contents -->

$<$ !ELEMENT repetition ((description)?, (olock_contents)*)>

$<$ !ATTLIST repetition

id CDATA \#IMPLIED

optional "false"

$\min \quad$ CDATA "O"

$\max$ CDATA "inf"

$>$ 


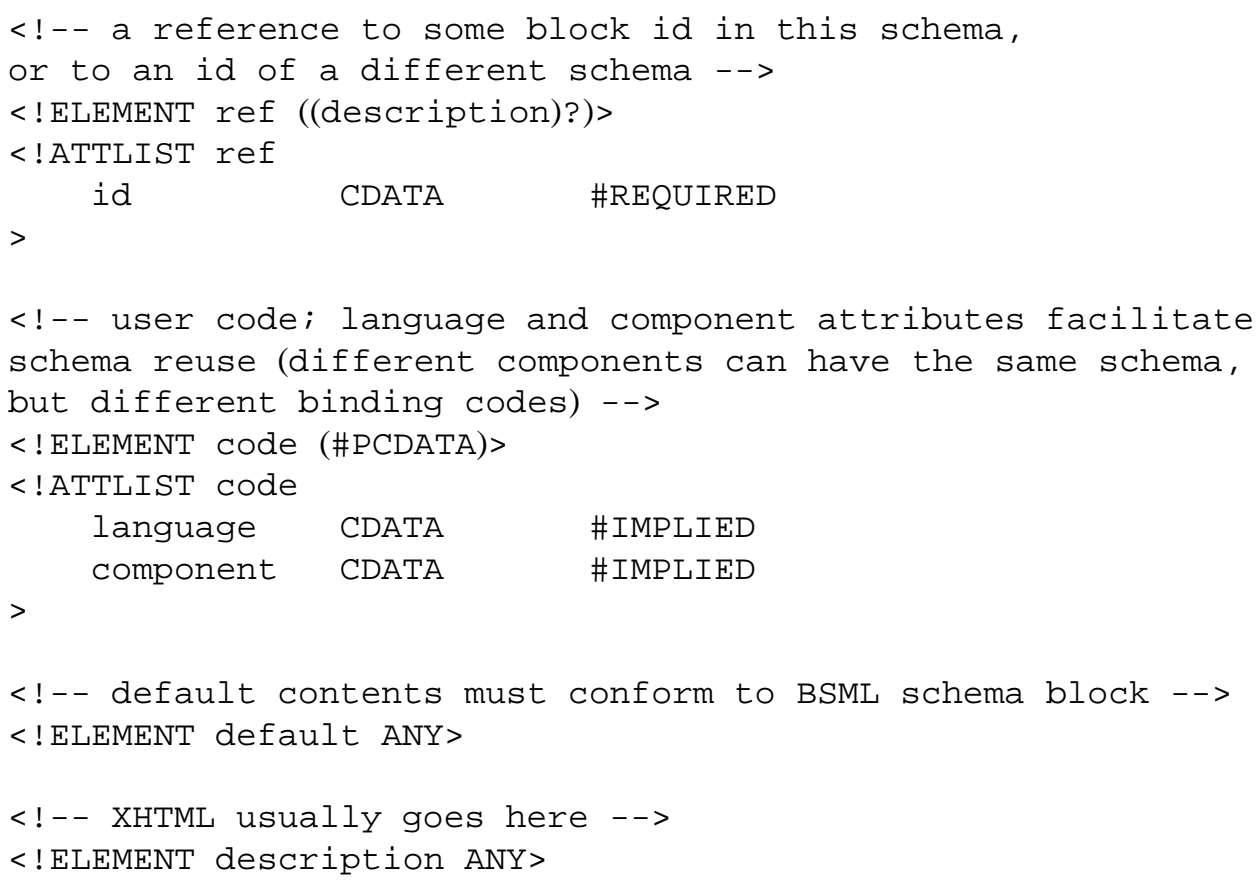




\section{References}

[1] N.R. Adam, I. Adiwijaya, T. Critchlow and R. Musick. Detecting Data and Schema Changes in Scientific Documents, in: Advances in Digital Libraries, 2000, pp. 160-172.

[2] V.S. Adve, R. Bagrodia, J.S. Browne, E. Deelman, A. Dube, E.N. Houstis, J.R. Rice, R. Sakellariou, D.J. Sundaram-Stukel, P.J. Teller and M.K. Vernon, POEMS: End-to-End Performance Design of Large Parallel Adaptive Computational Systems, IEEE Transactions on Software Engineering 26(11) (November 2000), 1027-1048.

[3] A.V. Aho, R. Sethi and J.D. Ullman, Compilers: Principles, Techniques and Tools, Addison-Wesley, 1986.

[4] R.G. Alscher, B.I. Chevone, L.S. Heath and N. Ramakrishnan, Expresso - A PSE for Bioinformatics: Finding Answers with Microarray Technology, in: Proceedings of the High Performance Computing Symposium, Advanced Simulation Technologies Conference, A. Tentner, ed., April 2001, pp. 64-69.

[5] J.B. Andersen, T.S. Rappaport and S. Yoshida, Propagation Measurements and Models for Wireless Communications Channels, IEEE Communications Magazine 33(1) (January 1995), 42-49.

[6] V. Apparao, S. Byrne, M. Champion, S. Isaacs, I. Jacobs, A. Le Hors, G. Nicol, J. Robie, R. Sutor, C. Wilson and L. Wood, Document Object Model (DOM) Level 1 Specification Version 1.0, W3C Recommendation Document, October 1998.

[7] W. Benger, H.-C. Hege, T. Radke and E. Seidel, Data Description via a Generalized Fiber Bundle Data Model, in: Tenth IEEE International Symposium on High Performance Distributed Computing, 2001.

[8] H.P. Bivens, Grid Workflow, Grid Computing Environments Working Group Document, Global Grid Forum, 2001.

[9] R. Bramley, K. Chiu, S. Diwan, D. Gannon, M. Govindaraju, N. Mukhi, B. Temko, and M. Yochuri, A Component Based Services Architecture for Building Distributed Applications, in: Proceedings of the Ninth IEEE International Symposium on High Performance Distributed Computing (HPDC'OO), IEEE Press, 2000.

[10] D. Brownell, SAX2, O'Reilly Books, January 2002.

[11] S. Chawathe and H. Garcia-Molina, Meaningful Change Detection in Structured Data, in: Proceedings of the ACMSIGMOD International Conference on Management of Data,
Tucson, Arizona, USA, 1997, pp. 26-37.

[12] J. Clark and M. Makoto, eds, RELAX NG Specification, OASIS Committee Specification Document, December 2001

[13] J. Clark, ed., XSL Transformations (XSLT) Version 1.0. W3C Recommendation Document, November 1999.

[14] T. Critchlow, M. Ganesh and R. Musick, Meta-Data Based Mediator Generation, in: Proceedings of the Third International Conference on Cooperative Information Systems, 1998, pp. $168-176$.

[15] T.T. Drashansky, E.N. Houstis, N. Ramakrishnan and J.R. Rice, Networked Agents for Scientific Computing, Communications of the ACM 42(3) (March 1999), 48-54.

[16] E. Gallopoulos, E.N. Houstis and J.R. Rice, Computer as Thinker/Doer: Problem-Solving Environments for Computational Science, IEEE Computational Science and Engineering 1(2) (1994), 11-23.

[17] A. Goel, C.A. Baker, C.A. Shaffer, B. Grossman, W.H. Mason, L.T. Watson and R.T. Haftka, VizCraft: A Problem-Solving Environment for Aircraft Configuration Design, IEEE/AIP Computing in Science and Engineering 3(1) (2001), 56-66.

[18] A. Goel, C. Phanouriou, F.A. Kamke, C.J. Ribbens, C.A. Shaffer and L.T. Watson, WBCSim: A Prototype Problem Solving Environment for Wood-Based Composites Simulation, Engineering with Computers 15 (1999), 198-210.

[19] D.R. Jones, C.D. Perttunen and B.E. Stuckman, Lipschitzian optimization without the Lipschitz Constant, Journal of Optimization Theory and Applications 79(1) (1993), 157-181.

[20] S. Markus, S. Weerawarana, E.N. Houstis and J.R. Rice, Scientific Computing via the World Wide Web: The Net PELLPACK PSE Server, IEEE Computational Science \& Engineering 4(3) (July-September 1997), 43-51.

[21] S. Pemberton, M. Altheim, D. Austin, F. Boumphrey, J. Burger, A.W. Donoho, S. Dooley, K. Hofrichter, P. Hoschka, M. Ishikawa, W. ten Tate, P. King, P. Klante, S. Matsui, S. McCarron, A. Navarro, Z. Nies, D. Raggett, P. Schmitz, S. Schnitzenbaumer, P. Stark, C. Wilson, T. Wugofski and D. Zigmond, XHTML 1.0: The Extensible HyperText Markup Language, W3C Recommendation Document, January 2000.

[22] J.R. Rice and R.F. Boisvert, From Scientific Software Libraries to Problem-Solving Environments, IEEE Computational Science \& Engineering 3(3) (Fall 1996), 44-53. 

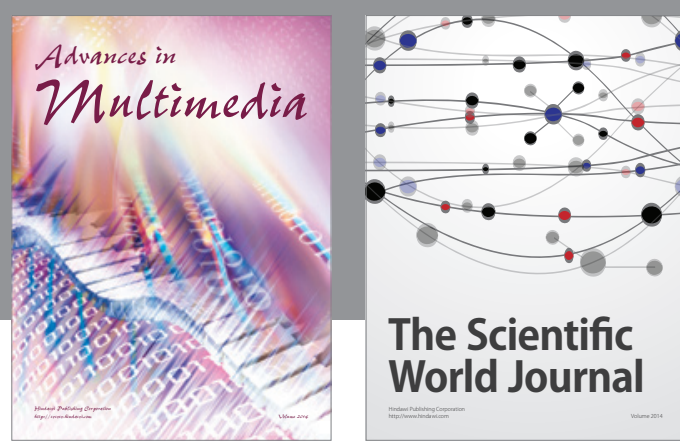

The Scientific World Journal
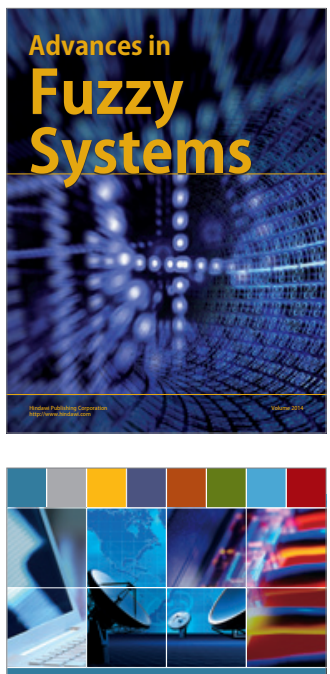

Computer Networks and Communications
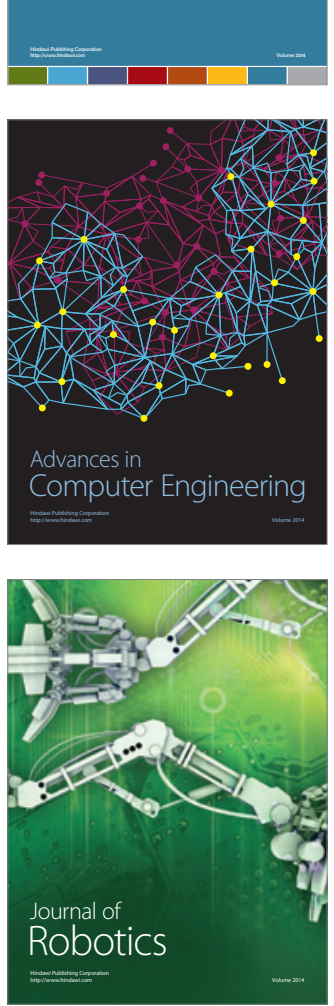
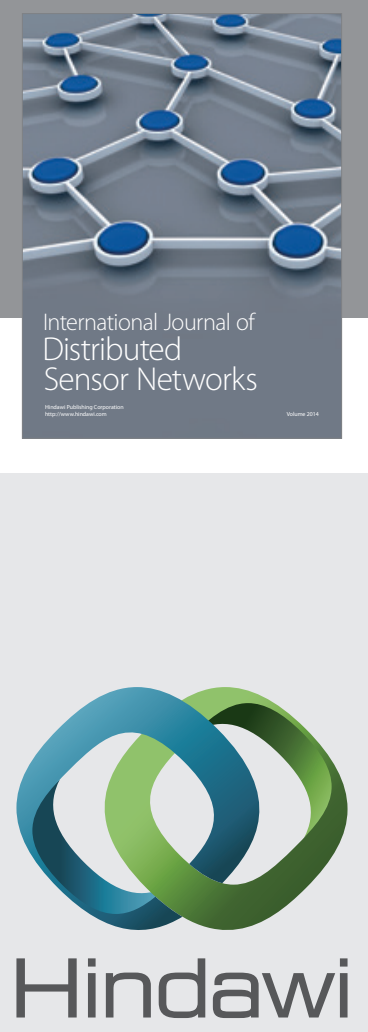

Submit your manuscripts at

http://www.hindawi.com
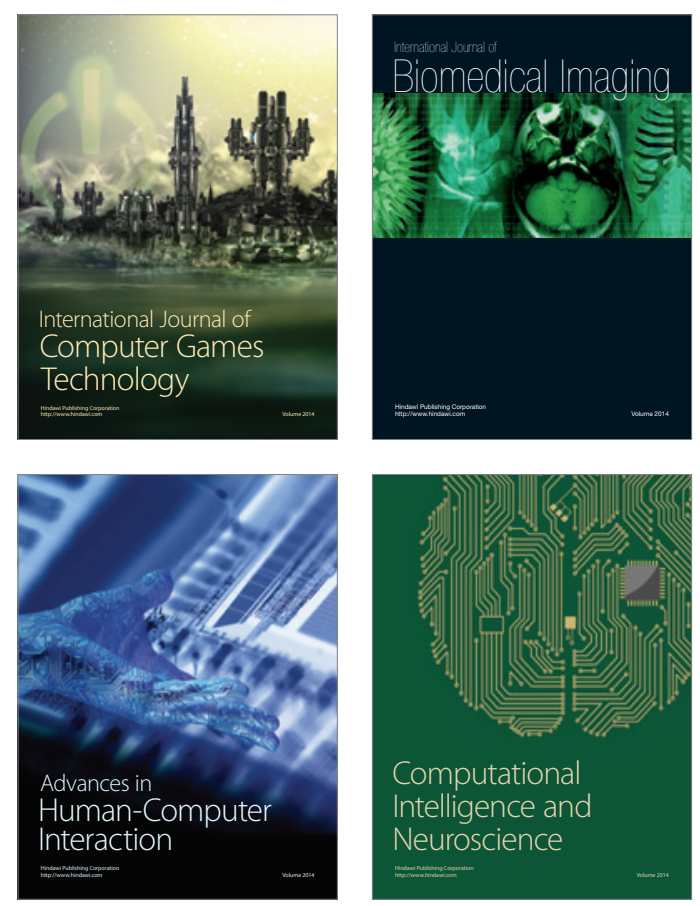
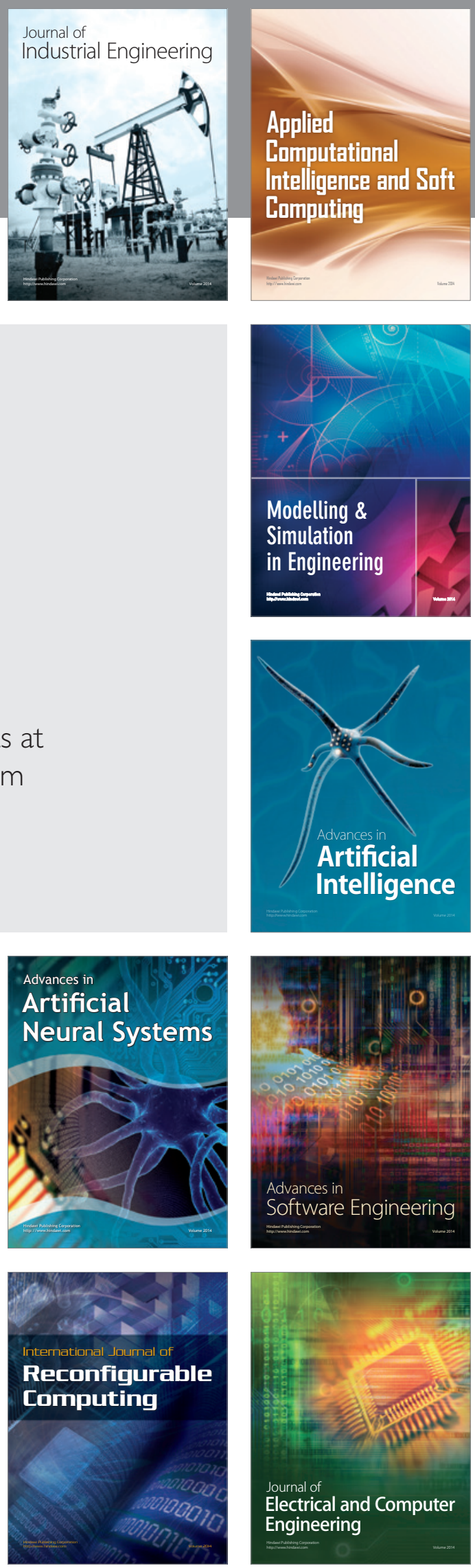